\title{
OPTIMAL CONTROL OF SEMILINEAR PARABOLIC EQUATIONS BY BV-FUNCTIONS*
}

\author{
EDUARDO $\mathrm{CASAS}^{\dagger}, \mathrm{FLORIAN} \mathrm{KRUSE}^{\ddagger}$, AND KARL KUNISCH ${ }^{\ddagger}$
}

\begin{abstract}
Optimal control problems for semilinear parabolic equations with control costs involving the total bounded variation seminorm are analyzed. This choice of control cost favors optimal controls which are piecewise constant and it penalizes the number of jumps. It is an appropriate choice if a simple structure of the optimal controls is desired, which, however, is still sufficiently flexible so that good tracking properties can be maintained. Existence of optimal controls, necessary and sufficient optimality conditions, and sparsity properties of the derivatives are obtained. Convergence of a finite element approximation is analyzed and numerical examples illustrating structural properties of the optimal controls are provided.
\end{abstract}

Key words. bounded variation functions, optimal control, sparsity, semilinear parabolic equations, first and second order optimality conditions, discontinuous Galerkin approximations

AMS subject classifications. 35K58, 49J20, 49J52, 49K20, 49M25

DOI. $10.1137 / 16 \mathrm{M} 1056511$

1. Introduction. This paper is dedicated to the analysis of the optimal control problem

(P) $\min _{u \in B V(0, T)^{m}} J(u)=\frac{1}{2}\left\|y_{u}-y_{d}\right\|_{L^{2}(Q)}^{2}+\sum_{j=1}^{m}\left(\alpha_{j}\left\|u_{j}^{\prime}\right\|_{\mathcal{M}(0, T)}+\frac{\beta_{j}}{2}\left(\int_{0}^{T} u_{j}(t) d t\right)^{2}\right)$,

where $u=\left(u_{j}\right)_{j=1}^{m}$ and $y_{u}$ is the solution to the parabolic state equation

$$
\left\{\begin{aligned}
\frac{\partial y}{\partial t}(x, t)-\Delta y(x, t)+f(x, t, y(x, t)) & =\sum_{j=1}^{m} u_{j} g_{j} & & \text { in } Q=\Omega \times(0, T), \\
y(x, t) & =0 & & \text { on } \Sigma=\Gamma \times(0, T), \\
y(x, 0) & =y_{0}(x) & & \text { in } \Omega .
\end{aligned}\right.
$$

Here, we assume that $\Omega$ is a bounded domain in $\mathbb{R}^{n}, 1 \leq n \leq 3$, with a Lipschitz boundary $\Gamma$, and $y_{0} \in L^{\infty}(\Omega)$. $B V(0, T)$ denotes the space of bounded variation functions defined in $(0, T)$, with $0<T<\infty$ given. The controllers in $(\mathrm{P})$ are supposed to be separable functions with respect to fixed spatial shape functions $g_{j}$ and free temporal amplitudes $u_{j}$. The specific new feature in $(\mathrm{P})$ is given by the choice of the control norm as the $\mathrm{BV}$-seminorm $\left\|u_{j}^{\prime}\right\|_{\mathcal{M}(0, T)}$. It enhances that the optimal controls are piecewise constant in time and that the number of jumps is penalized. The weights in $(\mathrm{P})$ are assumed to satisfy $\alpha_{j}>0$ and $\beta_{j} \geq 0$. Thus the goal of the optimal

${ }^{*}$ Received by the editors January 13, 2016; accepted for publication (in revised form) January 23, 2017; published electronically June 6, 2017.

http://www.siam.org/journals/sicon/55-3/M105651.html

Funding: The work of the first author was supported by Spanish Ministerio de Economía y Competitividad under project MTM2014-57531-P. The work of the third author was supported by the Austrian Science Fund (FWF) under grant SFB F32 (SFB "Mathematical Optimization and Applications in Biomedical Sciences").

${ }^{\dagger}$ Departamento de Matemática Aplicada y Ciencias de la Computación, E.T.S.I. Industriales y de Telecomunicación, Universidad de Cantabria, 39005 Santander, Spain (eduardo.casas@unican.es).

${ }^{\ddagger}$ Institute of Mathematics and Scientific Computing, University of Graz, Heinrichstrasse 36, A8010 Graz, Austria (florian.kruse@uni-graz.at, karl.kunisch@uni-graz.at). 
control problem $(\mathrm{P})$ is to achieve a simple control strategy while simultaneously being as close to the target $y_{d}$ as possible. Let us further comment on the importance of this fact. If we consider the classical formulation of the control problem with a quadratic cost functional for the control, then the optimal control $\bar{u}$ is equal to a multiple of the optimal adjoint state. Hence, while it is a regular function of time, its practical implementation can be involved in comparison to piecewise constant controls. Of course, $\bar{u}$ can be approximated by piecewise constant functions, but a good approximation may require many jumps. Looking for a simpler structure for $\bar{u}$, one can consider the bang-bang formulation of the control problem by introducing pointwise constraints on the control: $\alpha \leq u(t) \leq \beta$. Then, we can expect for $\bar{u}$ to take only the values $\alpha$ and $\beta$. A drawback of this approach is given by the fact that $\bar{u}$ frequently takes the extreme values all the time. This can lead to undesirable amounts of energy used to control the system. Our formulation pursues an optimal control $\bar{u}$ with a simple structure and with lower energy than in the bang-bang case: We look for a piecewise constant control with just a few jumps. Corollary 10 shows that this goal can be achieved with our formulation. The numerical tests also confirm the desired simple structure of the optimal controls. The use of the BV-seminorm necessitates to develop novel techniques for the analysis and numerical realization of (P).

The appearance of the mean $\int_{0}^{T} u_{j}(t) d t$ in the cost is related to the kernel of the BV-seminorm. For linear and certain classes of nonlinear functions $f$ the choice $\beta_{j}=0$ is admissible, while for more severe nonlinearities we have chosen the option $\beta_{j}>0$ to guarantee existence of a solution to $(\mathrm{P})$.

The choice of the control costs related to BV-norms or BV-seminorms has not received much attention in the literature. However, let us mention [10] where the effect of $\mathrm{L}^{2}$-, $\mathrm{H}^{1}$-, measure-valued, and BV-valued control costs on the qualitative behavior of the optimal control was pointed out and compared. In [13] the use of BVcosts was investigated further for the case of linear elliptic equations. BV-seminorm control costs are also employed in [5], where the control appears as a coefficient in the $p$-Laplace equation.

Let us also compare the use of the BV-term in $(\mathrm{P})$ with the efforts that have been made for studying optimal control problems with sparsity constraints. These formulations involve either measure-valued norms of the control or $L^{1}$-functionals combined with pointwise constraints on the control. We cite $[4,14]$ from among the many results which are now already available. Thus the use of the BV-seminorm can also be understood as a sparsity constraint for the first derivative, which in our case is the temporal derivative.

Let us briefly outline the following sections. Section 2 contains a precise problem statement, the analysis of the state equation, and the differentiability properties of the cost functional. The analysis of the optimal control problem, sparsity properties of the optimal controls as well as second order necessary and sufficient optimality conditions are contained in section 3. Section 4 is devoted to a finite element approximation of the control problem and its well-posedness. A convergence analysis of this approximation scheme is provided in section 5. In section 6 we derive an algorithm to solve the control problem. Numerical results illustrating that the desired behavior of the optimal controls can actually be observed numerically are presented in section 7 .

2. Assumptions and first consequences. We recall that a function $u \in$ $L^{1}(0, T)$ is a function of bounded variation if its distributional derivative $u^{\prime}$ belongs to the Banach space of real and regular Borel measures $\mathcal{M}(0, T)$. Given a measure 
$\mu \in \mathcal{M}(0, T)$, its norm is given by

$$
\|\mu\|_{\mathcal{M}(0, T)}=\sup \left\{\int_{0}^{T} z d \mu: z \in C_{0}(0, T) \text { and }\|z\|_{C_{0}(0, T)} \leq 1\right\}=|\mu|(0, T),
$$

where $C_{0}(0, T)$ denotes the Banach space of continuous functions $z:[0, T] \longrightarrow \mathbb{R}$ such that $z(0)=z(T)=0$, and $|\mu|$ is the total variation measure associated with $\mu$. On $B V(0, T)$ we consider the usual norm

$$
\|u\|_{B V(0, T)}=\|u\|_{L^{1}(0, T)}+\left\|u^{\prime}\right\|_{\mathcal{M}(0, T)}
$$

which makes $B V(0, T)$ a Banach space; see [1, Chapter 3] or [12, Chapter 1] for details. In what follows, we will denote

$$
a_{u}=\frac{1}{T} \int_{0}^{T} u(t) d t \text { and } \hat{u}=u-a_{u} \text { for every } u \in B V(0, T)
$$

By using [1, Theorem 3.44] it is easy to deduce that there exists a constant $C_{T}$ such that

$$
\|u\|:=\left|a_{u}\right|+\left\|u^{\prime}\right\|_{\mathcal{M}(0, T)} \leq \max (1, T)\|u\|_{B V(0, T)} \leq C_{T}\|u\|
$$

In addition, we mention that $B V(0, T)$ is the dual space of a separable Banach space. Therefore, every bounded sequence $\left\{u_{k}\right\}_{k=1}^{\infty}$ in $B V(0, T)$ has a subsequence converging weakly* to some $u \in B V(0, T)$. The weak* convergence $u_{k} \stackrel{*}{*} u$ implies that $u_{k} \rightarrow u$ strongly in $L^{1}(0, T)$ and $u_{k}^{\prime} \stackrel{*}{\rightarrow} u^{\prime}$ in $\mathcal{M}(0, T)$; see [1, pp. 124-125]. We will also use that $B V(0, T)$ is continuously embedded in $L^{\infty}(0, T)$ and compactly embedded in $L^{p}(0, T)$ for every $p<+\infty$; see [1, Corollary 3.49]. From this property we deduce that the convergence $u_{k} \stackrel{*}{\rightarrow} u$ in $B V(0, T)$ implies that $u_{k} \rightarrow u$ strongly in every $L^{p}(0, T)$ for all $p<+\infty$.

In the functional $J, y_{d}$ is given in $L^{\hat{p}}(Q)$, where $\hat{p}>1+\frac{n}{2}$ if $n>1$, and $\hat{p} \geq 2$ if $n=1, \alpha_{j}>0$ and $\beta_{j} \geq 0$ for $1 \leq j \leq m$. Further, the functions $\left\{g_{j}\right\}_{j=1}^{m} \subset L^{\infty}(\Omega) \backslash\{0\}$ have pairwise disjoint supports $\omega_{j}=\operatorname{supp} g_{j}$. Finally, we assume that $f: Q \times \mathbb{R} \longrightarrow \mathbb{R}$ is a Borel function, of class $C^{2}$ with respect to the last variable, and satisfies for almost all $(x, t) \in Q$

(3) $f(\cdot, \cdot, 0) \in L^{\hat{p}}(Q)$,

(4) $\frac{\partial f}{\partial y}(x, t, y) \geq 0 \quad \forall y \in \mathbb{R}$,

$$
\begin{aligned}
& \forall M>0 \exists C_{M}:\left|\frac{\partial f}{\partial y}(x, t, y)\right|+\left|\frac{\partial^{2} f}{\partial y^{2}}(x, t, y)\right| \leq C_{M} \quad \forall|y| \leq M \\
& \left\{\begin{array}{l}
\forall M>0 \text { and } \forall \rho>0 \exists \varepsilon>0 \text { such that } \\
\left|\frac{\partial^{2} f}{\partial y^{2}}\left(x, t, y_{2}\right)-\frac{\partial^{2} f}{\partial y^{2}}\left(x, t, y_{1}\right)\right| \leq \rho \text { if }\left|y_{2}-y_{1}\right|<\varepsilon \text { and }\left|y_{1}\right|,\left|y_{2}\right| \leq M
\end{array}\right.
\end{aligned}
$$

Let us observe that if $f$ is an affine function, $f(x, t, y)=c_{0}(x, t) y+d_{0}(x, t)$, then (3)-(6) hold if $c_{0} \geq 0$ in $Q, c_{0} \in L^{\infty}(Q)$, and $d_{0} \in L^{\hat{p}}(Q)$.

By using these assumptions, the following theorem can be proved in a standard way; see, for instance, [2] or [23, Theorem 5.5]. 
Proposition 1. For every $u \in L^{p}(0, T)^{m}$, with $p>1$, the state equation (1) has a unique solution $y_{u} \in L^{\infty}(Q) \cap L^{2}\left(0, T ; H_{0}^{1}(\Omega)\right)$. In addition, for every $M>0$ there exists a constant $K_{M}$ such that

$$
\left\|y_{u}\right\|_{L^{\infty}(Q)}+\left\|y_{u}\right\|_{L^{2}\left(0, T ; H_{0}^{1}(\Omega)\right)} \leq K_{M} \quad \forall u \in L^{p}(0, T)^{m}:\|u\|_{L^{p}(0, T)^{m}} \leq M .
$$

In what follows, we will denote $Y=L^{\infty}(Q) \cap L^{2}\left(0, T ; H_{0}^{1}(\Omega)\right)$ and $S: L^{p}(0, T)^{m}$ $\longrightarrow Y$ the mapping associating to each control $u$ the corresponding state $S(u)=y_{u}$, with $p>1$. By the implicit function theorem, we deduce in the classical way the following result [7, Theorem 5.1].

Proposition 2. The mapping $S: L^{p}(Q)^{m} \longrightarrow Y$ is of class $C^{2}$. For all elements $u, v$, and $w$ of $L^{p}(0, T)^{m}$, the functions $z_{v}=S^{\prime}(u) v$ and $z_{v w}=S^{\prime \prime}(u)(v, w)$ are the solutions of the problems

$$
\left\{\begin{aligned}
\frac{\partial z}{\partial t}-\Delta z+\frac{\partial f}{\partial y}\left(x, t, y_{u}\right) z & =\sum_{j=1}^{m} v_{j} g_{j} & & \text { in } Q \\
z & =0 & & \text { on } \Sigma, \\
z(x, 0) & =0 & & \text { in } \Omega,
\end{aligned}\right.
$$

and

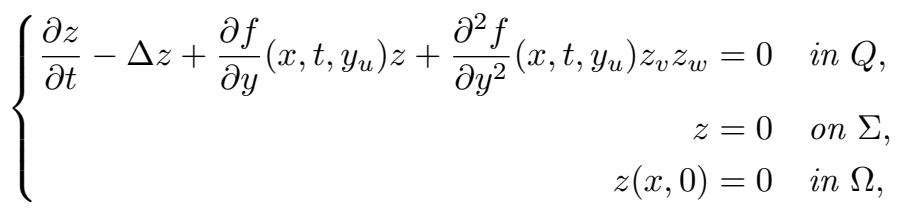

respectively.

Next, we analyze the differentiability of the cost functional. In $J$ we separate the smooth and the convex parts $J(u)=F(u)+G(u)$ with

$$
F(u)=\frac{1}{2}\left\|y_{u}-y_{d}\right\|_{L^{2}(Q)}^{2}+\sum_{j=1}^{m} \frac{\beta_{j}}{2}\left(\int_{0}^{T} u_{j}(t) d t\right)^{2} \text { and } G(u)=\sum_{j=1}^{m} \alpha_{j} g\left(u_{j}^{\prime}\right),
$$

where $g: \mathcal{M}(0, T) \longrightarrow \mathbb{R}$ is given by $g(\mu)=\|\mu\|_{\mathcal{M}(0, T)}$. From Proposition 2 and the chain rule the following proposition can be obtained.

Proposition 3. The functional $F: L^{p}(0, T)^{m} \longrightarrow \mathbb{R}$, with $p>1$, is of class $C^{2}$. The derivatives of $F$ are given by

$$
F^{\prime}(u) v=\sum_{j=1}^{m} \int_{0}^{T}\left(\int_{\omega_{j}} \varphi_{u}(x, t) g_{j}(x) d x+\beta_{j} \int_{0}^{T} u_{j}(t) d t\right) v_{j}(t) d t
$$

and

$$
F^{\prime \prime}(u)(v, w)=\int_{Q}\left(1-\varphi_{u} \frac{\partial^{2} f}{\partial y^{2}}\left(x, t, y_{u}\right)\right) z_{v} z_{w} d x d t+\sum_{j=1}^{m} \beta_{j} \int_{0}^{T} v_{j} d t \int_{0}^{T} w_{j} d t
$$

with $z_{v}=S^{\prime}(u) v, z_{w}=S^{\prime}(u) w$, and $\varphi_{u} \in Y \cap C(\bar{Q})$ is the adjoint state which satisfies

$$
\left\{\begin{aligned}
-\frac{\partial \varphi_{u}}{\partial t}-\Delta \varphi_{u}+\frac{\partial f}{\partial y}\left(x, t, y_{u}\right) \varphi_{u} & =y_{u}-y_{d} & & \text { in } Q, \\
\varphi_{u} & =0 & & \text { on } \Sigma, \\
\varphi_{u}(T) & =0 & & \text { in } \Omega .
\end{aligned}\right.
$$


The $L^{\infty}(Q)$ regularity of $\varphi_{u}$ follows from the assumptions on $y_{d}$ and the fact that $y_{u} \in L^{\infty}(Q)$. For the continuity of $\varphi_{u}$ in $\bar{Q}$ it is enough to use that the terminal and boundary conditions are zero.

Since $B V(0, T)^{m}$ is continuously embedded in $L^{\infty}(0, T)^{m}$, the mapping $F$ is well defined on $B V(0, T)^{m}$ and it is of class $C^{2}$.

Concerning the functional $g: \mathcal{M}(0, T) \longrightarrow \mathbb{R}, g(\mu)=\|\mu\|_{\mathcal{M}(0, T)}$, we note that it is Lipschitz continuous and convex. Hence, it has a subdifferential and a directional derivative, which are denoted by $\partial g(\mu)$ and $g^{\prime}(\mu ; \nu)$, respectively. The following propositions give some properties of $\partial g(\mu)$ and provide an expression for $g^{\prime}(\mu ; \nu)$.

Proposition 4 (see [6, Proposition 3.2]). If $\lambda \in \partial g(\mu)$ and $\lambda \in C_{0}(0, T)$, then we have $\|\lambda\|_{C_{0}(0, T)} \leq 1$. Moreover, if $\mu \neq 0$, the following properties hold:

1. $\|\lambda\|_{C_{0}(0, T)}=1$ and $\int_{0}^{T} \lambda d \mu=\|\mu\|_{\mathcal{M}(0, T)}$.

2. Taking the Jordan decomposition $\mu=\mu^{+}-\mu^{-}$, we have

$$
\begin{aligned}
& \operatorname{supp}\left(\mu^{+}\right) \subset\{t \in(0, T): \lambda(t)=+1\}, \\
& \operatorname{supp}\left(\mu^{-}\right) \subset\{t \in(0, T): \lambda(t)=-1\} .
\end{aligned}
$$

Before considering the directional derivative $g^{\prime}(\mu ; \nu)$, let us introduce some notation. Given two measures $\mu, \nu \in \mathcal{M}(0, T)$, we consider the Lebesgue decomposition of $\nu=\nu_{a}+\nu_{s}$ with respect to $|\mu|$, where $\nu_{a}$ is the absolutely continuous part of $\nu$ with respect to $|\mu|$, and $\nu_{s}$ is the singular part. Now, we take the Radon-Nikodym derivative of $\nu_{a}$ with respect to $|\mu|, d \nu_{a}=h_{\nu} d|\mu|$. Then we have

$$
\|\nu\|_{\mathcal{M}(0, T)}=\left\|\nu_{a}\right\|_{\mathcal{M}(0, T)}+\left\|\nu_{s}\right\|_{\mathcal{M}(0, T)}=\int_{0}^{T}\left|h_{\nu}\right| d|\mu|+\left\|\nu_{s}\right\|_{\mathcal{M}(0, T)}
$$

In particular, it is obvious that $\mu$ is absolutely continuous with respect to $|\mu|$. Consequently, we can express $d \mu=h d|\mu|$, where $h$ is measurable with respect to $|\mu|$ and $|h(x)|=1$ for all $x \in(0, T), d \mu^{+}=h^{+} d|\mu|$, and $d \mu^{-}=h^{-} d|\mu|$, where $\mu=\mu^{+}-\mu^{-}$is the Jordan decomposition of $\mu$. See, for instance, [20, Chapter 6] for details.

Proposition 5 (see [6, Proposition 3.3]). Let $\mu, \nu \in \mathcal{M}(0, T)$, then

$$
g^{\prime}(\mu ; \nu)=\int_{0}^{T} h_{\nu} d \mu+\left\|\nu_{s}\right\|_{\mathcal{M}(0, T)} .
$$

Now, we analyze the mapping $G$. To this end, let us introduce the operator $D_{t}: B V(0, T) \longrightarrow \mathcal{M}(0, T)$ by $D_{t} u=u^{\prime}$. Its adjoint operator is defined by

$$
D_{t}^{*}: \mathcal{M}(0, T)^{*} \longrightarrow B V(0, T)^{*}, \quad\left\langle D_{t}^{*} \lambda, u\right\rangle_{B V(0, T)^{*}, B V(0, T)}=\left\langle\lambda, u^{\prime}\right\rangle_{\mathcal{M}(0, T)^{*}, \mathcal{M}(0, T)}
$$

Proposition 6. The following identities hold for all $u \in B V(0, T)$ :

$$
\begin{aligned}
& \partial\left(g \circ D_{t}\right)(u)=D_{t}^{*} \partial g\left(u^{\prime}\right), \\
& \left(g \circ D_{t}\right)^{\prime}(u ; v)=\int_{0}^{T} h_{v^{\prime}} d u^{\prime}+\left\|v_{s}^{\prime}\right\|_{\mathcal{M}(0, T)},
\end{aligned}
$$

where $d v^{\prime}=h_{v^{\prime}} d\left|u^{\prime}\right|+d v_{s}^{\prime}$ is the Lebesgue decomposition of $v^{\prime}$ with respect to $\left|u^{\prime}\right|$.

Proof. Since $g: \mathcal{M}(0, T) \longrightarrow \mathbb{R}$ is convex and continuous and $D_{t}: B V(0, T) \longrightarrow$ $\mathcal{M}(0, T)$ is a linear and continuous mapping, we can apply the chain rule [11, Chapter I, Proposition 5.7] to deduce that $\partial\left(g \circ D_{t}\right)(u)=D_{t}^{*} \partial g\left(u^{\prime}\right)$, which immediately leads to (15). 
To verify (16) it is enough to observe that

$$
\left(g \circ D_{t}\right)^{\prime}(u ; v)=g^{\prime}\left(u^{\prime} ; v^{\prime}\right)
$$

and to apply (14). This completes the proof.

3. Analysis of the optimal control problem (P). This section is devoted to the proof of the existence of at least one solution of $(\mathrm{P})$ and to the optimality conditions and their consequences.

THEOREM 7. Let us assume that one of the following assumptions hold:

1. $\beta_{j}>0$ for every $1 \leq j \leq m$.

2. There exist $q \in[1,2)$ and $C>0$ such that

$$
\frac{\partial f}{\partial y}(x, t, y) \leq C\left(1+|y|^{q}\right) \text { for a.a. }(x, t) \in Q .
$$

Then, problem $(\mathrm{P})$ has at least one solution. Moreover, if $f$ is affine with respect to $y$, the solution is unique.

Let us observe that condition (17) is satisfied in the case of affine functions with respect to $y$.

Proof. Let $\left\{u_{k}\right\}_{k=1}^{\infty} \subset B V(0, T)^{m}$ be a minimizing sequence. We prove that this sequence is bounded in $B V(0, T)^{m}$. As introduced in section 2, we consider the decomposition $u_{k}=a_{k}+\hat{u}_{k}$, where $a_{k}=\left(a_{k, 1}, \ldots, a_{k, m}\right), \hat{u}_{k}=\left(\hat{u}_{k, 1}, \ldots, \hat{u}_{k, m}\right)$, and

$$
a_{k}=\frac{1}{T} \int_{0}^{T} u_{k}(t) d t \quad \text { and } \quad \hat{u}_{k}=u_{k}-a_{k} .
$$

Since

$$
\begin{aligned}
& \sum_{j=1}^{m}\left(\alpha_{j}\left\|\hat{u}_{k, j}^{\prime}\right\|_{\mathcal{M}(0, T)}+\frac{\beta_{j}}{2} a_{k j}^{2}\right) \\
& =\sum_{j=1}^{m}\left(\alpha_{j}\left\|u_{k j}^{\prime}\right\|_{\mathcal{M}(0, T)}+\frac{\beta_{j}}{2}\left(\int_{0}^{T} u_{k j}(t) d t\right)^{2}\right) \leq J\left(u_{k}\right) \leq J(0)<+\infty,
\end{aligned}
$$

taking into account (2), we deduce that $\left\{\hat{u}_{k}\right\}_{k=1}^{\infty}$ is bounded in $B V(0, T)^{m}$. Now we prove the boundedness of $\left\{a_{k}\right\}_{k=1}^{\infty}$ in $\mathbb{R}^{m}$. This boundedness is obvious if the first assumption is satisfied. Otherwise, let us denote by $y_{k}$ and $\hat{y}_{k}$ the solutions (1) associated to the controls $u_{k}$ and $\hat{u}_{k}$, respectively. From the inequalities

$$
\frac{1}{2}\left\|y_{k}-y_{d}\right\|_{L^{2}(Q)}^{2} \leq J\left(u_{k}\right) \leq J(0)<+\infty
$$

we infer the boundedness of $\left\{y_{k}\right\}_{k=1}^{\infty}$ in $L^{2}(Q)$. Moreover, the boundedness of $\left\{\hat{u}_{k}\right\}_{k=1}^{\infty}$ in $B V(0, T)^{m}$ and (7) imply that $\left\{\hat{y}_{k}\right\}_{k=1}^{\infty}$ is also bounded in $L^{2}(Q)$. Now, we define $z_{k}=y_{k}-\hat{y}_{k}$, which produces a bounded sequence in $L^{2}(Q)$ as well. Subtracting the equations satisfied by $y_{k}$ and $\hat{y}_{k}$ and using the mean value theorem, we infer that

$$
\left\{\begin{aligned}
\frac{\partial z_{k}}{\partial t}-\Delta z_{k}+\frac{\partial f}{\partial y}\left(x, t, \xi_{k}\right) z_{k} & =\sum_{j=1}^{m} a_{k, j} g_{j} & & \text { in } Q, \\
z_{k} & =0 & & \text { on } \Sigma, \\
z_{k}(x, 0) & =0 & & \text { in } \Omega,
\end{aligned}\right.
$$


where $\xi_{k}(x, t)=\hat{y}_{k}(x, t)+\theta_{k}(x, t)\left(y_{k}(x, t)-\hat{y}_{k}(x, t)\right)=\hat{y}_{k}(x, t)+\theta_{k}(x, t) z_{k}(x, t)$ with $0 \leq \theta_{k}(x, t) \leq 1$. To argue by contradiction, let us assume that

$$
\rho_{k}=\max _{1 \leq j \leq m}\left|a_{k, j}\right| \rightarrow+\infty \text { as } k \rightarrow \infty .
$$

Then, introducing $\zeta_{k}=\frac{1}{\rho_{k}} z_{k}$, we obtain from (18) that

$$
\left\{\begin{aligned}
\frac{\partial \zeta_{k}}{\partial t}-\Delta \zeta_{k}+\frac{\partial f}{\partial y}\left(x, t, \xi_{k}\right) \zeta_{k} & =\frac{1}{\rho_{k}} \sum_{j=1}^{m} a_{k, j} g_{j} & & \text { in } Q, \\
\zeta_{k} & =0 & & \text { on } \Sigma, \\
\zeta_{k}(x, 0) & =0 & & \text { in } \Omega .
\end{aligned}\right.
$$

From this equation, using (4), (5), and the boundedness of the right-hand side in $L^{\infty}(Q)$, we have that $\left\|\zeta_{k}\right\|_{L^{\infty}(Q)} \leq M$ for some $M>0$ and all $k$. Moreover, the boundedness of $\left\{z_{k}\right\}_{k=1}^{\infty}$ in $L^{2}(Q)$ implies that $\left\|\zeta_{k}\right\|_{L^{2}(Q)} \rightarrow 0$. Now, (17) and Hölder's inequality with $\frac{2}{q}$ and $\frac{2}{2-q}$ lead to

$$
\begin{aligned}
& \int_{Q}\left|\frac{\partial f}{\partial y}\left(x, t, \xi_{k}\right) \zeta_{k}\right| d x d t \leq C\left(\int_{Q}\left(1+\left|\xi_{k}\right|^{q}\right)^{\frac{2}{q}} d x d t\right)^{\frac{q}{2}}\left(\int_{Q}\left|\zeta_{k}\right|^{\frac{2}{2-q}} d x d t\right)^{\frac{2-q}{q}} \\
& \leq C\left(\int_{Q}\left(1+\left[\left|\hat{y}_{k}\right|+\left|z_{k}\right|\right]^{q}\right)^{\frac{2}{q}} d x d t\right)^{\frac{q}{2}}\left(\int_{Q}\left|\zeta_{k}\right|^{2} d x d t\right)^{\frac{2-q}{q}}\left\|\zeta_{k}\right\|_{L^{\infty}(Q)}^{\frac{2 q-2}{q}} \rightarrow 0 .
\end{aligned}
$$

Combined with the aforementioned properties of $\left\{\zeta_{k}\right\}_{k=1}^{\infty}$ this shows that the left-hand side of the partial differential equation in (19) converges to zero in the distribution sense. However, by the definition of $\rho_{k}$ we have that the right-hand side does not converge to zero, which is a contradiction. Consequently, $\left\{a_{k}\right\}_{k=1}^{\infty}$ is a bounded sequence in $\mathbb{R}^{m}$, hence the minimizing sequence $\left\{u_{k}\right\}_{k=1}^{\infty}$ is bounded in $B V(0, T)^{m}$ because of (2). Therefore, we can take a subsequence, denoted in the same way, such that $u_{k} *{ }^{*} \bar{u}$ in $B V(0, T)^{m}$, which implies $u_{k} \rightarrow \bar{u}$ strongly in $L^{p}(0, T)^{m}$ for every $p<+\infty$. As a consequence of Proposition 2 we have that $y_{k} \rightarrow \bar{y}$ strongly in $\mathrm{Y}$, where $\bar{y}$ is the state associated to $\bar{u}$, and thus $F\left(u_{k}\right) \rightarrow F(\bar{u})$. Furthermore, the convergence $u_{k, j}^{\prime} \stackrel{*}{\rightarrow} \bar{u}_{k, j}^{\prime}$ in $\mathcal{M}(0, T)$ for every $1 \leq j \leq m$ yields that

$$
G(\bar{u})=\sum_{j=1}^{m} \alpha_{j}\left\|\bar{u}_{j}^{\prime}\right\|_{\mathcal{M}(0, T)} \leq \liminf _{k \rightarrow \infty} \sum_{j=1}^{m} \alpha_{j}\left\|u_{k, j}^{\prime}\right\|_{\mathcal{M}(0, T)}=\liminf _{k \rightarrow \infty} G\left(u_{k}\right) .
$$

Hence, $J(\bar{u}) \leq \liminf _{k \rightarrow \infty} J\left(u_{k}\right)=\inf (\mathrm{P})$ and $\bar{u}$ is a solution of $(\mathrm{P})$.

The uniqueness of a solution when $f$ is affine with respect to $y$ is an immediate consequence of the strict convexity of $F$ and the convexity of $G$.

Next, we analyze the first order optimality conditions. Since $(\mathrm{P})$ is not a convex problem it is convenient to deal with local solutions.

Definition 8. Let $\bar{u} \in B V(0, T)^{m}$. We shall call $\bar{u}$ a local solution of $(\mathrm{P})$ if there exists $\varepsilon>0$ such that

$$
J(\bar{u}) \leq J(u) \quad \forall u \in B V(0, T)^{m}:\|u-\bar{u}\|_{B V(0, T)^{m}} \leq \varepsilon .
$$

We say that $\bar{u}$ is an $L^{p}(0, T)^{m}$-local solution $(1 \leq p \leq \infty)$ if the above statement is true with the $L^{p}(0, T)^{m}$ norm in place of the $B V(0, T)^{m}$ norm. Finally, $\bar{u}$ is called a 
strong local solution if

$$
J(\bar{u}) \leq J(u) \quad \forall u \in B V(0, T)^{m}:\left\|y_{u}-\bar{y}\right\|_{L^{\infty}(Q)} \leq \varepsilon
$$

for some $\varepsilon>0$, where $\bar{y}$ and $y_{u}$ denote the states associated to $\bar{u}$ and $u$, respectively. The solution is said to be strict in any of the previous senses if the inequality $J(\bar{u})<$ $J(u)$ holds in the above statements whenever $\bar{u} \neq u$.

We have the following relationships among these concepts. Since $B V(0, T)$ is continuously embedded into $L^{p}(0, T)$ for any $p \in[1,+\infty]$, we deduce that if $\bar{u}$ is an $L^{p}(0, T)^{m}$-local solution of $(\mathrm{P})$, then it is a local solution. On the other hand, from Propositions 1 and 2 we infer that any strong local solution is an $L^{p}(0, T)^{m}$-local solution for $1<p \leq+\infty$.

Given $\bar{u} \in B V(0, T)^{m}$ with associated state and adjoint state $\bar{y}$ and $\bar{\varphi}$, respectively, we define

$$
\bar{\Phi}_{j}(t)=\int_{0}^{t} \int_{\omega_{j}} \bar{\varphi}(x, s) g_{j}(x) d x d s+\beta_{j} t \int_{0}^{T} \bar{u}_{j}(s) d s, \quad 1 \leq j \leq m .
$$

This quantity will allow us to obtain information on the structure of the optimal control $\bar{u}$. From Corollary 10 below we shall deduce that the support of $\bar{u}_{j}^{\prime}$ is contained in the set where $\left|\bar{\Phi}_{j}(t)\right|=\alpha_{j}$. In particular, jumps in $\bar{u}_{j}$ can only occur at $t$ with $\left|\bar{\Phi}_{j}(t)\right|=\alpha_{j}$. But at first we need to derive the following structure theorem for $\bar{\Phi}_{j}$.

TheOrem 9. If $\bar{u}$ is a local solution of $(\mathrm{P})$, then $\bar{\Phi}_{j} \in C^{1}[0, T] \cap C_{0}(0, T)$ for $1 \leq j \leq m$ and they satisfy

$$
\begin{aligned}
& \left\|\bar{\Phi}_{j}\right\|_{C_{0}(0, T)} \begin{cases}=\alpha_{j} & \text { if } \bar{u}_{j}^{\prime} \neq 0, \\
\leq \alpha_{j} & \text { if } \bar{u}_{j}^{\prime}=0,\end{cases} \\
& \int_{0}^{T} \bar{\Phi}_{j} d \bar{u}_{j}^{\prime}=\left\|\bar{\Phi}_{j}\right\|_{C_{0}(0, T)}\left\|\bar{u}_{j}^{\prime}\right\|_{\mathcal{M}(0, T)} .
\end{aligned}
$$

Proof. From Proposition 3 we know that $\bar{\varphi} \in C(\bar{Q})$, hence $\bar{\Phi}_{j} \in C^{1}[0, T]$ follows for every $j$. Let us fix one component $j$ and denote by $e_{j}$ the $j$ th unit vector of the canonical basis in $\mathbb{R}^{m}$. Given $u \in B V(0, T)$, from the local optimality of $\bar{u}$ and the convexity of $G$ we deduce for every $0<\rho<1$ small enough

$$
\begin{aligned}
& 0 \leq \frac{J\left(\bar{u}+\rho u e_{j}\right)-J(\bar{u})}{\rho}=\frac{F\left(\bar{u}+\rho u e_{j}\right)-F(\bar{u})}{\rho}+\frac{G\left(\bar{u}+\rho u e_{j}\right)-G(\bar{u})}{\rho} \\
& \leq \frac{F\left(\bar{u}+\rho u e_{j}\right)-F(\bar{u})}{\rho}+\left[G\left(\bar{u}+u e_{j}\right)-G(\bar{u})\right] \\
& =\frac{F\left(\bar{u}+\rho u e_{j}\right)-F(\bar{u})}{\rho}+\alpha_{j}\left[\left(g \circ D_{t}\right)\left(\bar{u}_{j}+u\right)-g\left(\bar{u}_{j}^{\prime}\right)\right] .
\end{aligned}
$$

Passing to the limit as $\rho \rightarrow 0$ in the above inequality and using (10) we obtain for every $u \in B V(0, T)$

$0 \leq \int_{0}^{T}\left(\int_{\omega_{j}} \bar{\varphi}(x, t) g_{j}(x) d x+\beta_{j} \int_{0}^{T} \bar{u}_{j}(s) d s\right) u_{j}(t) d t+\alpha_{j}\left[\left(g \circ D_{t}\right)\left(\bar{u}_{j}+u\right)-g\left(\bar{u}_{j}^{\prime}\right)\right]$. 
Using (20), the above inequality can be written as

$$
-\frac{1}{\alpha_{j}} \int_{0}^{T} \bar{\Phi}_{j}^{\prime}(t) u(t) d t+g\left(\bar{u}_{j}^{\prime}\right) \leq\left(g \circ D_{t}\right)\left(\bar{u}_{j}+u\right) \quad \forall u \in B V(0, T) .
$$

From the above inequality, the definition of the subdifferential of a convex function, and using (15) it follows that

$$
-\frac{1}{\alpha_{j}} \bar{\Phi}_{j}^{\prime} \in \partial\left(g \circ D_{t}\right)\left(\bar{u}_{j}\right)=D_{t}^{*} \partial g\left(\bar{u}_{j}^{\prime}\right) .
$$

Therefore, there exists $\bar{\lambda}_{j} \in \partial g\left(\bar{u}_{j}^{\prime}\right) \subset \mathcal{M}(0, T)^{*}$ such that

$$
-\frac{1}{\alpha_{j}} \int_{0}^{T} \bar{\Phi}_{j}^{\prime}(t) u(t) d t=\left\langle\bar{\lambda}_{j}, u^{\prime}\right\rangle \quad \forall u \in B V(0, T) .
$$

A first consequence of this identity is that $\Phi_{j}(T)=0$. Indeed, it is enough to take $u \equiv 1$ and use that $\bar{\Phi}_{j}(0)=0$, which follows obviously from the definition.

Given $u \in B V(0, T)$, we can select a sequence $\left\{u_{k}\right\}_{k=1}^{\infty} \subset C^{\infty}[0, T]$ converging weakly* to $u$ in $B V(0, T)$; see [1, Remark 3.22]. Using this fact and the property $\bar{\Phi}_{j}(T)=\bar{\Phi}_{j}(0)=0$, we observe

$$
-\int_{0}^{T} \bar{\Phi}_{j}^{\prime}(t) u(t) d t=-\lim _{k \rightarrow \infty} \int_{0}^{T} \bar{\Phi}_{j}^{\prime}(t) u_{k}(t) d t=\lim _{k \rightarrow \infty} \int_{0}^{T} \bar{\Phi}_{j}(t) u_{k}^{\prime}(t) d t=\left\langle u^{\prime}, \bar{\Phi}_{j}\right\rangle .
$$

Since this identity holds for all $u \in B V(0, T)$, and any measure in $\mathcal{M}(0, T)$ is the derivative of a function of $B V(0, T)$, we infer from $(24)$ that $\bar{\lambda}_{j}=\frac{1}{\alpha_{j}} \bar{\Phi}_{j} \in C_{0}(0, T)$. Thus we have that $\frac{1}{\alpha_{j}} \bar{\Phi}_{j} \in \partial g\left(\bar{u}_{j}^{\prime}\right)$, which means

$$
\left\langle\mu-\bar{u}_{j}^{\prime}, \frac{1}{\alpha_{j}} \bar{\Phi}_{j}\right\rangle+\left\|\bar{u}_{j}^{\prime}\right\|_{\mathcal{M}(0, T)} \leq\|\mu\|_{\mathcal{M}(0, T)} \quad \forall \mu \in \mathcal{M}(0, T) .
$$

Taking $\mu=2 \bar{u}_{j}^{\prime}$ and $\mu=\frac{1}{2} \bar{u}_{j}^{\prime}$, respectively, we deduce that

$$
\left\langle\bar{u}_{j}^{\prime}, \frac{1}{\alpha_{j}} \bar{\Phi}_{j}\right\rangle=\left\|\bar{u}_{j}^{\prime}\right\|_{\mathcal{M}(0, T)}
$$

and consequently,

$$
\left\langle\mu, \frac{1}{\alpha_{j}} \bar{\Phi}_{j}\right\rangle \leq\|\mu\|_{\mathcal{M}(0, T)} \quad \forall \mu \in \mathcal{M}(0, T)
$$

The last two relationships are equivalent to (21) and (22).

COROLLARY 10. Under the assumptions of Theorem 9, the following inclusions are valid for each $j \in\{1, \ldots, m\}$ for which $\bar{u}_{j}$ is not a constant function on $[0, T]$ :

$$
\left\{\begin{array}{l}
\operatorname{supp}\left(\bar{u}_{j}^{\prime+}\right) \subset\left\{t \in[0, T]: \bar{\Phi}_{j}(t)=+\alpha_{j}\right\} \\
\operatorname{supp}\left(\bar{u}_{j}^{\prime-}\right) \subset\left\{t \in[0, T]: \bar{\Phi}_{j}(t)=-\alpha_{j}\right\}
\end{array}\right.
$$

where $\bar{u}_{j}^{\prime}=\bar{u}_{j}^{\prime+}-\bar{u}_{j}^{\prime-}$ is the Jordan decomposition of the measure $\bar{u}_{j}^{\prime}$. 
This corollary is a straightforward consequence of (21), (22), Proposition 4 with $\lambda=-\frac{1}{\alpha_{j}} \bar{\Phi}_{j}$, and the fact that $\bar{u}_{j}^{\prime} \neq 0$ if $\bar{u}_{j}$ is not a constant function in $[0, T]$.

Remark 11. 1. Let us observe that if there are only finitely many $t$ with $\bar{\Phi}_{j}(t) \in$ $\left\{-\alpha_{j},+\alpha_{j}\right\}$, then $\bar{u}_{j}^{\prime}$ is a combination of Dirac measures centered at those points. In particular, we obtain that $\bar{u}_{j}$ is piecewise constant in $[0, T]$. This will be illustrated in the numerical examples; cf. sections 7.1 and 7.2.

2. Given $\alpha=\left(\alpha_{j}\right)_{j=1}^{m}$, let us denote by $\bar{u}_{\alpha}=\left(\bar{u}_{\alpha, j}\right)_{j=1}^{m}$ a solution of (P) and by $\left(\bar{y}_{\alpha}, \bar{\varphi}_{\alpha}\right)$ the associated state and adjoint state. We note that if $\alpha_{j}$ is decreased, then the $B V(0, T)$ seminorm of $\bar{u}_{\alpha, j}$ is increasing. On the contrary, if $\alpha_{j}$ is increased, then the $B V(0, T)$ seminorm of $\bar{u}_{\alpha, j}$ is decreasing. In fact, there is a threshold $M_{j}<+\infty$ such that if $\alpha_{j}>M_{j}$, then $\bar{u}_{\alpha, j}^{\prime}=0$, i.e., $\bar{u}_{\alpha, j}$ is constant in $[0, T]$. Moreover, there exists a vector $\bar{\xi} \in \mathbb{R}^{m}$ such that for any $\alpha$ with $\alpha_{j}>M_{j}$ for all $1 \leq j \leq m$, the constant function $\bar{\xi}$ is a solution of $(\mathrm{P})$. Let us provide an upper bound for these values $M_{j}$.

Let $y^{0}$ be the solution of the state equation associated to the control $u \equiv 0$. From the optimality of $\bar{u}_{\alpha}$ we get

$$
\frac{1}{2}\left\|\bar{y}_{\alpha}-y_{d}\right\|_{L^{2}(Q)}^{2}+\sum_{j=1}^{m} \frac{\beta_{j}}{2}\left(\int_{0}^{T} \bar{u}_{\alpha, j}(t) d t\right)^{2} \leq J\left(\bar{u}_{\alpha}\right) \leq J(0)=\frac{1}{2}\left\|y^{0}-y_{d}\right\|_{L^{2}(Q)}^{2} .
$$

From these inequalities we deduce

$$
\left\|\bar{y}_{\alpha}-y_{d}\right\|_{L^{2}(Q)} \leq\left\|y^{0}-y_{d}\right\|_{L^{2}(Q)} \quad \text { and } \quad \beta_{j}\left|\int_{0}^{T} \bar{u}_{\alpha, j}(t) d t\right| \leq \sqrt{\beta_{j}}\left\|y^{0}-y_{d}\right\|_{L^{2}(Q)} .
$$

From the adjoint state equation we obtain

$$
\left\|\bar{\varphi}_{\alpha}\right\|_{L^{\infty}\left(0, T ; L^{2}(\Omega)\right)} \leq C_{\Omega}\left\|\bar{y}_{\alpha}-y_{d}\right\|_{L^{2}(Q)} \leq C_{\Omega}\left\|y^{0}-y_{d}\right\|_{L^{2}(Q)},
$$

where $C_{\Omega}$ is the constant satisfying $\|z\|_{L^{2}(\Omega)} \leq C_{\Omega}\|\nabla z\|_{L^{2}(\Omega)}$ for any $z \in H_{0}^{1}(\Omega)$. From the definition of $\bar{\Phi}_{j}$ and the above estimates we get for every $t \in[0, T]$

$$
\begin{aligned}
& \left|\bar{\Phi}_{j}(t)\right| \leq T\left\|\bar{\varphi}_{\alpha}\right\|_{L^{\infty}\left(0, T ; L^{2}(\Omega)\right)}\left\|g_{j}\right\|_{L^{2}\left(\omega_{j}\right)}+\beta_{j}\left|\int_{0}^{T} \bar{u}_{\alpha, j}(t) d t\right| \\
& \leq\left(T C_{\Omega}\left\|g_{j}\right\|_{L^{2}\left(\omega_{j}\right)}+\sqrt{\beta_{j}}\right)\left\|y^{0}-y_{d}\right\|_{L^{2}(Q)}=M_{j} .
\end{aligned}
$$

Relations (25) imply that $\bar{u}_{\alpha, j}^{\prime}=0$ if $\alpha_{j}>M_{j}$.

To prepare for the second order necessary conditions we introduce the critical cone as follows

$$
C_{\bar{u}}=\left\{v \in B V(0, T)^{m}: F^{\prime}(\bar{u}) v+G^{\prime}(\bar{u} ; v)=0\right\} .
$$

It seems natural that the second order optimality conditions must be imposed only on those directions where the directional derivatives vanish. Let us point out some properties of this critical cone.

Proposition 12. $C_{\bar{u}}$ is a closed convex cone that can equivalently be expressed in the form

$$
C_{\bar{u}}=\left\{v \in B V(0, T)^{m}: \int_{0}^{T} \bar{\Phi}_{j}(t) d v_{j s}^{\prime}(t)=\alpha_{j}\left\|v_{j s}^{\prime}\right\|_{\mathcal{M}(0, T)}, 1 \leq j \leq m\right\},
$$

where $v_{j s}^{\prime}$ is the singular part of the measure $v_{j}^{\prime}$ with respect to $\left|\bar{u}_{j}^{\prime}\right|$. 
The identity (27) shows that the criterion for $v$ to be in $C_{\bar{u}}$ can be expressed in terms of the singular part of $v_{j}^{\prime}$ with respect to $\left|\bar{u}_{j}^{\prime}\right|$ for $1 \leq j \leq m$. In particular, any function $v \in B(0, T)^{m}$ such that $v_{j}^{\prime}$ is absolutely continuous with respect to $\left|\bar{u}_{j}^{\prime}\right|$ for every $j$ is an element of the critical cone.

Proof. The cone property and closedness of $C_{\bar{u}}$ are a straightforward consequence of the continuity and positive homogeneity of the mapping $v \rightarrow F^{\prime}(\bar{u}) v+G^{\prime}(\bar{u} ; v)$. Let us prove the convexity property. First, we observe that (10) and the definition of $\bar{\Phi}_{j}$ implies that

$$
F^{\prime}(\bar{u}) v=\sum_{j=1}^{m} \int_{0}^{T} \bar{\Phi}_{j}^{\prime}(t) v_{j}(t) d t \quad \forall v \in B V(0, T)^{m} .
$$

Taking into account (23), using the definition of the subdifferential and passing to the limit as $\rho \searrow 0$ we infer for $1 \leq j \leq m$

$$
-\frac{1}{\alpha_{j}} \int_{0}^{T} \bar{\Phi}_{j}^{\prime}(t) v_{j}(t) d t \leq \frac{g\left(\bar{u}_{j}^{\prime}+\rho v_{j}^{\prime}\right)-g\left(\bar{u}_{j}^{\prime}\right)}{\rho} \rightarrow g^{\prime}\left(\bar{u}_{j}^{\prime} ; v_{j}^{\prime}\right) .
$$

Multiplying this inequality by $\alpha_{j}$ and summing in $j$ we get with (28)

$$
F^{\prime}(\bar{u}) v+G^{\prime}(\bar{u} ; v) \geq 0 \quad \forall v \in B V(0, T)^{m} .
$$

Therefore, $v \in C_{\bar{u}}$ if and only if $F^{\prime}(\bar{u}) v+G^{\prime}(\bar{u} ; v) \leq 0$. Since the mapping $v \in$ $B V(0, T)^{m} \rightarrow F^{\prime}(\bar{u}) v+G^{\prime}(\bar{u} ; v)$ is convex, we conclude the convexity of $C_{\bar{u}}$.

From (28), making an integration by parts as in the proof of Theorem 9, and using the Lebesgue decomposition $d v_{j}^{\prime}=h_{v_{j}^{\prime}} d\left|\bar{u}_{j}^{\prime}\right|+d v_{j s}^{\prime}$, we obtain

$$
F^{\prime}(\bar{u}) v=-\sum_{j=1}^{m} \int_{0}^{T} \bar{\Phi}_{j} d v_{j}^{\prime}=-\left\{\sum_{j=1}^{m} \int_{0}^{T} \bar{\Phi}_{j} h_{v_{j}^{\prime}} d\left|\bar{u}_{j}^{\prime}\right|+\int_{0}^{T} \bar{\Phi}_{j} d v_{j s}^{\prime}\right\} .
$$

From (25) we deduce that $d\left|\bar{u}_{j}^{\prime}\right|=\frac{1}{\alpha_{j}} \bar{\Phi}_{j} d \bar{u}_{j}^{\prime}$ for $1 \leq j \leq m$. Inserting this identity in the above equality we infer

$$
F^{\prime}(\bar{u}) v=-\left\{\sum_{j=1}^{m} \alpha_{j} \int_{0}^{T} h_{v_{j}^{\prime}} d \bar{u}_{j}^{\prime}+\int_{0}^{T} \bar{\Phi}_{j} d v_{j s}^{\prime}\right\} .
$$

Now, using (16) it follows that

$$
G^{\prime}(\bar{u} ; v)=\sum_{j=1}^{m} \alpha_{j}\left\{\int_{0}^{T} h_{v_{j}^{\prime}} d \bar{u}_{j}^{\prime}+\left\|v_{j s}^{\prime}\right\|_{\mathcal{M}(0, T)}\right\} .
$$

This equality and (30) lead to

$$
F^{\prime}(\bar{u}) v+G^{\prime}(\bar{u} ; v)=\sum_{j=1}^{m}\left\{-\int_{0}^{T} \bar{\Phi}_{j} d v_{j s}^{\prime}+\alpha_{j}\left\|v_{j s}^{\prime}\right\|_{\mathcal{M}(0, T)}\right\},
$$

which is equivalent to the expressions given in (27) for $1 \leq j \leq m$.

Now we formulate the second order necessary optimality conditions. 
Theorem 13. If $\bar{u}$ is a local minimum of $(\mathrm{P})$, then $F^{\prime \prime}(\bar{u}) v^{2} \geq 0$ for all $v \in C_{\bar{u}}$.

Proof. Let $v$ be an element in $C_{\bar{u}}$ and consider the Lebesgue decomposition $d v_{j}^{\prime}=$ $h_{v_{j}^{\prime}} d\left|\bar{u}_{j}^{\prime}\right|+d v_{j s}^{\prime}, 1 \leq j \leq m$. For every integer $k \geq 1$ we set

$$
h_{j, k}(t)=\operatorname{proj}_{[-k,+k]}\left(h_{v_{j}^{\prime}}(t)\right) \quad \text { and } \quad d v_{j, k}^{\prime}=h_{j, k} d\left|\bar{u}_{j}^{\prime}\right|+d v_{j s}^{\prime} .
$$

Let us take $v_{j, k} \in L^{1}(0, T)$ as the primitive of $v_{j, k}^{\prime}$ with $\int_{0}^{T}\left(v_{j}-v_{j, k}\right) d t=0$, and set $v_{k}=\left(v_{1, k}, \ldots, v_{m, k}\right)$. Then, we have $\left\|v_{j}^{\prime}-v_{j, k}^{\prime}\right\|_{\mathcal{M}(0, T)}=\left\|h_{v_{j}^{\prime}}-h_{j, k}\right\|_{L^{1}\left(\left|\bar{u}_{j}^{\prime}\right|\right)} \rightarrow 0$ by Lebesgue's dominated convergence theorem. Hence $v_{k} \rightarrow v$ in $B V(0, T)^{m}$. Moreover, since the singular parts of $v_{j, k}^{\prime}$ and $v_{j}^{\prime}$ with respect to $\left|\bar{u}_{j}^{\prime}\right|$ coincide and $v \in C_{\bar{u}}$, then (27) implies that $v_{k} \in C_{\bar{u}}$ for every $k$.

For any $0<\rho<\frac{1}{k}$, using (13) and (14), we find

$$
\begin{aligned}
& \frac{G\left(\bar{u}+\rho v_{k}\right)-G(\bar{u})}{\rho}=\sum_{j=1}^{m} \alpha_{j} \frac{g\left(\bar{u}_{j}^{\prime}+\rho v_{j, k}^{\prime}\right)-g\left(\bar{u}_{j}^{\prime}\right)}{\rho} \\
& =\sum_{j=1}^{m} \alpha_{j}\left\{\int_{0}^{T} \frac{\left|1+\rho h_{v_{j, k}^{\prime}}\right|-1}{\rho} d\left|\bar{u}_{j}^{\prime}\right|+\left\|v_{j s}^{\prime}\right\|_{\mathcal{M}(0, T)}\right\} \\
& =\sum_{j=1}^{m} \alpha_{j}\left\{\int_{0}^{T} \frac{\left|1+\rho h_{v_{j, k}^{\prime}}\right|-1}{\rho} d \bar{u}_{j}^{\prime+}+\int_{0}^{T} \frac{\left|-1+\rho h_{v_{j, k}^{\prime}}\right|-1}{\rho} d \bar{u}_{j}^{\prime-}+\left\|v_{j s}^{\prime}\right\|_{\mathcal{M}(0, T)}\right\} \\
& =\sum_{j=1}^{m} \alpha_{j}\left\{\int_{0}^{T} h_{v_{j, k}^{\prime}} d \bar{u}_{j}^{\prime}+\left\|v_{j s}^{\prime}\right\|_{\mathcal{M}(0, T)}\right\}=G^{\prime}\left(\bar{u} ; v_{k}\right) .
\end{aligned}
$$

Using that $\bar{u}$ is a local minimum of $J$ and making a Taylor expansion we obtain for every $k$ and $0<\rho<\frac{1}{k}$ the existence of $\theta=\theta(k, \rho)$, with $0<\theta<1$, such that

$0 \leq \frac{J\left(\bar{u}+\rho v_{k}\right)-J(\bar{u})}{\rho}=F^{\prime}(\bar{u}) v_{k}+\frac{\rho}{2} F^{\prime \prime}\left(\bar{u}+\theta \rho v_{k}\right) v_{k}^{2}+G^{\prime}\left(\bar{u} ; v_{k}\right)=\frac{\rho}{2} F^{\prime \prime}\left(\bar{u}+\theta \rho v_{k}\right) v_{k}^{2}$,

since $v_{k} \in C_{\bar{u}}$. Finally, dividing the last term by $\rho / 2$ and taking the limit for $\rho \rightarrow 0$ and subsequently for $k \rightarrow \infty$, we arrive at $F^{\prime \prime}(\bar{u}) v^{2} \geq 0$.

As usual we have to consider an extended cone of critical directions to formulate a sufficient second order condition for optimality. For every $\tau>0$ we denote

$$
C_{\bar{u}}^{\tau}=\left\{v \in B V(0, T)^{m}: F^{\prime}(\bar{u}) v+G^{\prime}(\bar{u} ; v) \leq \tau\left(\left\|z_{v}\right\|_{L^{2}(Q)}+\sum_{j=1}^{m} \beta_{j}\left|\int_{0}^{T} v_{j}(t) d t\right|\right)\right\},
$$

where $z_{v}=S^{\prime}(\bar{u}) v$, with $S$ defined just above Proposition 2. The second order condition involves this cone as follows:

(SSOC) There exist positive constants $\kappa$ and $\tau$ such that

$$
F^{\prime \prime}(\bar{u}) v^{2} \geq \kappa\left\|z_{v}\right\|_{L^{2}(Q)}^{2} \quad \forall v \in C_{\bar{u}}^{\tau} .
$$

TheOrem 14. Let $\bar{u} \in B V(0, T)^{m}$ satisfy the first order optimality conditions (21)-(22) and (SSOC). Then, there exist positive constants $\varepsilon>0$ and $\nu>0$ such that

$$
J(\bar{u})+\frac{\nu}{2}\left\|z_{u-\bar{u}}\right\|_{L}^{2}(Q)^{2} \leq J(u) \forall u \in B V(0, T)^{m}:\left\|y_{u}-\bar{y}\right\|_{L^{\infty}(Q)} \leq \varepsilon .
$$


The proof of this theorem can be done along the lines of [8, Theorem 9]. Let us point out some small differences. First, the parameter $\gamma$ in [8] must be taken as zero. Second, we have a nondifferentiable part in the cost functional and a slightly different cone of critical directions. To deal with the nondifferentiable term $G$ we use (29) and its convexity and Lipschitz continuity: for every $u \in B V(0, T)^{m}$,

$$
\begin{aligned}
& J(u)-J(\bar{u})=F^{\prime}(\bar{u})(u-\bar{u})+\frac{1}{2} F^{\prime \prime}(\bar{u}+\theta(u-\bar{u}))(u-\bar{u})^{2}+G(u)-G(\bar{u}) \\
& \geq \frac{1}{2} F^{\prime \prime}(\bar{u}+\theta(u-\bar{u}))(u-\bar{u})^{2}+F^{\prime}(\bar{u})(u-\bar{u})+G^{\prime}(\bar{u} ; u-\bar{u}) \\
& \geq \frac{1}{2} F^{\prime \prime}(\bar{u}+\theta(u-\bar{u}))(u-\bar{u})^{2} .
\end{aligned}
$$

In this way we eliminate the nondifferentiable part of the cost functional. The rest is the same.

COROLlaRY 15. Under the assumptions of Theorem 14 there exist two constants $\varepsilon>0$ and $\delta>0$ such that

$$
J(\bar{u})+\frac{\delta}{2}\left\|y_{u}-\bar{y}\right\|_{L^{2}(Q)}^{2} \leq J(u) \forall u \in B V(0, T)^{m}:\left\|y_{u}-\bar{y}\right\|_{L^{\infty}(Q)} \leq \varepsilon .
$$

This is an immediate consequence of (32) and the estimate

$$
\left\|y_{u}-\bar{y}\right\|_{L^{2}(Q)} \leq M\left\|z_{u-\bar{u}}\right\|_{L^{2}(Q)} \quad \forall u \in B V(0, T)^{m}:\left\|y_{u}-\bar{y}\right\|_{L^{\infty}(Q)} \leq \varepsilon ;
$$

see $[8$, Corollary 3] for the proof.

We observe that the sufficient second order optimality condition (31) along with the first order optimality condition imply that $\bar{u}$ is a strong local solution of $(\mathrm{P})$.

4. Approximation of the control problem. In this section we assume that $\Omega$ is a convex set and $y_{0} \in L^{\infty}(\Omega) \cap H_{0}^{1}(\Omega)$. Then, it is well known that the solutions $y_{u}$ of (1) belong to $C\left([0, T], H_{0}^{1}(\Omega)\right) \cap H^{2,1}(Q)$; see, for instance, [21, Proposition 2.4].

We consider a $\mathrm{dG}(0) \mathrm{cG}(1)$ discontinuous Galerkin approximation of the state equation (1), i.e., piecewise constant in time and linear nodal basis finite elements in space; see, e.g., [22]. Let $\left\{\mathcal{K}_{h}\right\}_{h>0}$ be a quasi-uniform family of triangulations of $\bar{\Omega}$; see [9]. We set $\bar{\Omega}_{h}=\cup_{K \in \mathcal{K}_{h}} K$ with $\Omega_{h}$ and $\Gamma_{h}$ being its interior and boundary, respectively. We assume that the vertices of $\mathcal{K}_{h}$ placed on the boundary $\Gamma_{h}$ are also points of $\Gamma$ and there exists a constant $C_{\Gamma}>0$ such that $\operatorname{dist}(x, \Gamma) \leq C_{\Gamma} h^{2}$ for every $x \in \Gamma_{h}$. This always holds if $\Gamma$ is a $C^{2}$ boundary and $n=2$. In the case of polygonal or polyhedral domains it is reasonable to assume that the triangulation satisfies $\Gamma_{h}=\Gamma$, hence this condition obviously holds. This also holds if $n=1$. From this assumption we know [19, section 5.2] that

$$
\left|\Omega \backslash \Omega_{h}\right| \leq C h^{2}
$$

where $|\cdot|$ denotes the Lebesgue measure.

We also introduce a temporal grid $0=t_{0}<t_{1}<\cdots<t_{N_{\tau}}=T$ with $\tau_{k}=t_{k}-t_{k-1}$ and set $\tau=\max _{1 \leq k \leq N_{\tau}} \tau_{k}$. We denote $I_{k}=\left(t_{k-1}, t_{k}\right)$. We assume that there exist $\rho_{T}>0$ such that $\tau \leq \rho_{T} \tau_{k}$ for $1 \leq k \leq N_{\tau}$. We will use the notation $\sigma=(h, \tau)$ and $Q_{h}=\Omega_{h} \times(0, T)$. 
4.1. Discretization of the controls. Associated with the grid $\left\{t_{k}\right\}_{k=0}^{N_{\tau}}$ we define the subspace

$$
U_{\tau}=\left\{u_{\tau} \in B V(0, T): u_{\tau}=\sum_{k=1}^{N_{\tau}} u_{k} \chi_{k}, \text { with }\left\{u_{k}\right\}_{k=1}^{N_{\tau}} \subset \mathbb{R}\right\},
$$

where $\chi_{k}$ denotes the characteristic function of the interval $I_{k}$. Let us observe that the elements $u_{\tau} \in U_{\tau}$ are piecewise constant functions whose distributional derivative is given by

$$
u_{\tau}^{\prime}=D_{t} u_{\tau}=\sum_{k=2}^{N_{\tau}}\left(u_{k}-u_{k-1}\right) \delta_{t_{k-1}} \quad \text { and } \quad\left\|u_{\tau}^{\prime}\right\|_{\mathcal{M}(0, T)}=\sum_{k=2}^{N_{\tau}}\left|u_{k}-u_{k-1}\right|,
$$

where $\delta_{t}$ denotes the Dirac measure concentrated at the point $t$. We further define the projection operator

$$
\Lambda_{\tau}: B V(0, T) \longrightarrow U_{\tau}, \quad \Lambda_{\tau} u=\sum_{k=1}^{N_{\tau}}\left(\frac{1}{\tau_{k}} \int_{I_{k}} u(t) d t\right) \chi_{k} .
$$

Proposition 16. For any $u \in B V(0, T)$ the following properties hold:

$$
\begin{aligned}
& \left\|u-\Lambda_{\tau} u\right\|_{L^{1}(0, T)} \leq \tau\left\|D_{t} u\right\|_{\mathcal{M}(0, T)}, \\
& \left\|D_{t} \Lambda_{\tau} u\right\|_{\mathcal{M}(0, T)} \leq\left\|D_{t} u\right\|_{\mathcal{M}(0, T)}, \\
& \lim _{\tau \rightarrow 0}\left\|D_{t} \Lambda_{\tau} u\right\|_{\mathcal{M}(0, T)}=\left\|D_{t} u\right\|_{\mathcal{M}(0, T)} .
\end{aligned}
$$

Proof. The inequality (36) is simple to establish for $u \in C^{1}[0, T]$. Henceforth, let $u \in B V(0, T)$. Then there exists a sequence $\left\{u_{j}\right\}_{j=1}^{\infty} \subset C^{\infty}[0, T]$ such that

$$
\left\|u-u_{j}\right\|_{L^{1}(0, T)}+||\left|D_{t} u\left\|_{\mathcal{M}(0, T)}-\right\| D_{t} u_{j} \|_{\mathcal{M}(0, T)}\right| \leq \frac{1}{j} \quad \forall j \geq 1 ;
$$

see [1, Remark 3.22]. Now we estimate as follows:

$$
\begin{gathered}
\left\|u-\Lambda_{\tau} u\right\|_{L^{1}(0, T)} \leq\left\|u-u_{j}\right\|_{L^{1}(0, T)}+\left\|u_{j}-\Lambda_{\tau} u_{j}\right\|_{L^{1}(0, T)}+\left\|\Lambda_{\tau} u_{j}-\Lambda_{\tau} u\right\|_{L^{1}(0, T)} \\
\leq\left\|u-u_{j}\right\|_{L^{1}(0, T)}+\tau\left\|D_{t} u_{j}\right\|_{\mathcal{M}(0, T)}+\left\|u_{j}-u\right\|_{L^{1}(0, T)} \leq \frac{2}{j}+\tau\left\|D_{t} u_{j}\right\|_{\mathcal{M}(0, T)} .
\end{gathered}
$$

Using (39) we can pass to the limit in the above inequality as $j \rightarrow \infty$ to deduce (36).

Let us prove (37). First, we assume again that $u \in C^{\infty}[0, T]$. From the continuity of $u$ and the mean value theorem for integrals we deduce the existence of points $\xi_{k} \in I_{k}, 1 \leq k \leq N_{\tau}$, such that

$$
\Lambda_{\tau} u=\sum_{k=1}^{N_{\tau}} u\left(\xi_{k}\right) \chi_{k}
$$

Then we have with (35)

$$
\begin{aligned}
& \left\|D_{t} \Lambda_{\tau} u\right\|_{\mathcal{M}(0, T)}=\sum_{k=2}^{N_{\tau}}\left|u\left(\xi_{k}\right)-u\left(\xi_{k-1}\right)\right| \\
& \leq \sum_{k=2}^{N_{\tau}} \int_{\xi_{k-1}}^{\xi_{k}}\left|u^{\prime}(t)\right| d t \leq \int_{0}^{T}\left|u^{\prime}(t)\right| d t=\left\|D_{t} u\right\|_{\mathcal{M}(0, T)} .
\end{aligned}
$$


For the case $u \in B V(0, T)$, we take again a sequence $\left\{u_{j}\right\}_{j=1}^{\infty} \subset C^{\infty}[0, T]$ satisfying (39). The convergence $u_{j} \rightarrow u$ in $L^{1}(0, T)$ obviously implies that $\Lambda_{\tau} u_{j} \rightarrow \Lambda_{\tau} u$ in $L^{1}(0, T)$. Then, using [1, Proposition 3.6], inequality (37) for every $u_{j}$, and (39) we conclude

$$
\left\|D_{t} \Lambda_{\tau} u\right\|_{\mathcal{M}(0, T)} \leq \liminf _{j \rightarrow \infty}\left\|D_{t} \Lambda_{\tau} u_{j}\right\|_{\mathcal{M}(0, T)} \leq \liminf _{j \rightarrow \infty}\left\|D_{t} u_{j}\right\|_{\mathcal{M}(0, T)}=\left\|D_{t} u\right\|_{\mathcal{M}(0, T)}
$$

which implies (37).

Finally, to prove (38) we use (36), [1, Proposition 3.6] and (37) to obtain

$$
\left\|D_{t} u\right\|_{\mathcal{M}(0, T)} \leq \liminf _{\tau \rightarrow 0}\left\|D_{t} \Lambda_{\tau} u\right\|_{\mathcal{M}(0, T)} \leq \limsup _{\tau \rightarrow 0}\left\|D_{t} \Lambda_{\tau} u\right\|_{\mathcal{M}(0, T)} \leq\left\|D_{t} u\right\|_{\mathcal{M}(0, T)} .
$$

This completes the proof.

4.2. Discrete state equation. Associated with the interior nodes of the triangulation $\left\{x_{j}\right\}_{j=1}^{N_{h}}$ we consider the space

$$
Y_{h}=\left\{y_{h} \in C_{0}(\Omega): y_{h}=\sum_{j=1}^{N_{h}} y_{j} e_{j} \text { with }\left\{y_{j}\right\}_{j=1}^{N_{h}} \subset \mathbb{R}\right\}
$$

where $\left\{e_{j}\right\}_{j=1}^{N_{h}}$ is the nodal basis formed by the continuous piecewise linear functions such that $e_{j}\left(x_{i}\right)=\delta_{i j}$ for every $1 \leq i, j \leq N_{h}$. For every $\sigma$ we define the space of discrete states by

$$
\mathcal{Y}_{\sigma}=\left\{y_{\sigma} \in L^{2}\left(I, Y_{h}\right):\left.y_{\sigma}\right|_{I_{k}} \in Y_{h}, 1 \leq k \leq N_{\tau}\right\} .
$$

The elements $y_{\sigma} \in \mathcal{Y}_{\sigma}$ can be represented in the form

$$
y_{\sigma}=\sum_{k=1}^{N_{\tau}} y_{k, h} \chi_{k}=\sum_{k=1}^{N_{\tau}} \sum_{j=1}^{N_{h}} y_{k j} \chi_{k} e_{j} \text { with }\left\{y_{k, h}\right\}_{k=1}^{N_{\tau}} \subset Y_{h} \text { and }\left\{y_{k j}\right\}_{\substack{1 \leq k \leq N_{\tau} \\ 1 \leq j \leq N_{h}}} \subset \mathbb{R}
$$

We approximate the state equation (1) as follows. For any control $u \in B V(0, T)^{m}$ we define the associated discrete state $y_{\sigma} \in \mathcal{Y}_{\sigma}$ as the solution of the system

$$
\left\{\begin{array}{l}
\left(\frac{y_{k, h}-y_{k-1, h}}{\tau_{k}}, z_{h}\right)+a\left(y_{k, h}, z_{h}\right)+\frac{1}{\tau_{k}} \int_{I_{k}}\left(f\left(\cdot, t, y_{k, h}\right), z_{h}\right) d t \\
=\frac{1}{\tau_{k}} \sum_{j=1}^{m}\left(g_{j}, z_{h}\right) \int_{I_{k}} u_{j}(t) d t \quad \forall z_{h} \in Y_{h}, 1 \leq k \leq N_{\tau}, \\
y_{0, h}=y_{0 h},
\end{array}\right.
$$

where $(\cdot, \cdot)$ denotes the scalar product in $L^{2}(\Omega), a$ is the bilinear form associated to the operator $-\Delta$, i.e.,

$$
a(y, z)=\int_{\Omega} \nabla y \cdot \nabla z d x
$$

and $y_{0 h}$ is the projection $P_{h} y_{0}$ of $y_{0}$ on $Y_{h}$ given by the variational equation

$$
\left(P_{h} y_{0}, z_{h}\right)=\left(y_{0}, z_{h}\right) \quad \forall z_{h} \in Y_{h} .
$$

It is well known that $y_{0 h} \rightarrow y_{0}$ in $H_{0}^{1}(\Omega)$. 
Proposition 17. For every $u \in B V(0, T)^{m}$ the system (41) has a unique solution $y_{\sigma} \in \mathcal{Y}_{\sigma}$. In addition, if either $f$ is affine with respect to the state or if $n<3$, then the following estimate holds:

$$
\left\|y_{u}-y_{\sigma}\right\|_{L^{2}(Q)} \leq C\left(\tau+h^{2}\right),
$$

where $C$ is independent of $\sigma$.

Remark 18. These results are proved in [16] and [17] for $f$ affine and nonlinear, respectively. The constant $C$ there depends on the norms of the state in $H^{2,1}(Q)$, and also on the $L^{\infty}(Q)$ norm in the semilinear case. These quantities can be estimated in our case by the $L^{2}(0, T)^{m}$ norm of $u$. During the preparation of this manuscript the following result was proved by Boris Vexler. Assuming that $\tau \leq C_{0} h^{\theta}$ for some $C_{0}>0$ and $\theta>0$, and $y_{0} \in H^{2}(\Omega) \cap H_{0}^{1}(\Omega)$, then the estimate

$$
\left\|y_{u}-y_{\sigma}\right\|_{L^{2}(Q)} \leq C\left(\tau+\left(\log \frac{T}{\tau}\right)^{2} h^{2}\right)
$$

holds.

Remark 19. Given $\left\{u_{j}\right\}_{j=1}^{m} \subset B V(0, T)$, we observe that

$$
\int_{I_{k}} u_{j}(t) d t=\int_{I_{k}} \Lambda_{\tau} u_{j}(t) d t \quad \forall 1 \leq j \leq m \quad \text { and } \quad 1 \leq \mathrm{k} \leq \mathrm{N}_{\tau}
$$

Utilizing this in (41), we deduce that the discrete states associated to $\left\{u_{j}\right\}_{j=1}^{m}$ and $\left\{\Lambda_{\tau} u_{j}\right\}_{j=1}^{m}$ coincide.

4.3. Discrete optimal control problem. The discrete control problem is defined as

$$
\left(\mathrm{P}_{\sigma}\right) \min _{u \in B V(0, T)^{m}} J_{\sigma}(u)=\frac{1}{2}\left\|y_{\sigma}-y_{d}\right\|_{L^{2}\left(Q_{h}\right)}^{2}+\sum_{j=1}^{m}\left(\alpha_{j}\left\|u_{j}^{\prime}\right\|_{\mathcal{M}(0, T)}+\frac{\beta_{j}}{2}\left(\int_{0}^{T} u_{j}(t) d t\right)^{2}\right),
$$

where $y_{\sigma}$ is the discrete state associated to $u=\left(u_{j}\right)_{j=1}^{m}$.

The following assumption will be used to analyze the existence and uniqueness of a solution of $\left(\mathrm{P}_{\sigma}\right)$ :

(A) The mapping $z_{h} \in Y_{h} \longrightarrow\left(\left(g_{j}, z_{h}\right)\right)_{j=1}^{m} \in \mathbb{R}^{m}$ is surjective.

Lemma 20. There exists $h_{0}>0$ such that $(A)$ holds for every $h<h_{0}$.

Proof. Let us recall that $\left\{e_{k}\right\}_{k=1}^{N_{h}}$ denotes the nodal basis of $Y_{h}$. Since the supports $\omega_{j}$ of the functions $g_{j}$ are compact and disjoint, we deduce the existence of $\hat{h}>0$ such that for every $h<\hat{h}$, if for some $e_{k}$ and some $1 \leq j \leq m$ we have that $\operatorname{supp}\left(e_{k}\right) \cap \omega_{j} \neq \emptyset$, then $\operatorname{supp}\left(e_{k}\right) \cap \omega_{i}=\emptyset$ for every $i \neq j$.

Moreover, there exists $\tilde{h}$ with the following property: $\forall h<\tilde{h}$ and $\forall j$ there exists some $k$ such that $\left(g_{j}, e_{k}\right) \neq 0$. Indeed, if this is not the case, we infer the existence of a sequence $\left\{h_{i}\right\}_{i=1}^{\infty}$ decreasing to 0 such that $\left(g_{j}, z_{h_{i}}\right)=0$ for every $z_{h_{i}} \in Y_{h_{i}}$. In particular, taking $z_{h_{i}}$ equal to the $L^{2}(\Omega)$-projection of $g_{j}$ on $Y_{h_{i}}$ we obtain

$$
\left\|g_{j}\right\|_{L^{2}(\Omega)}^{2}=\lim _{i \rightarrow \infty}\left(g_{j}, z_{h_{i}}\right)=0
$$

which contradicts the assumption $g_{j} \neq 0$ imposed for $(\mathrm{P})$. 
Finally, for any $h<h_{0}=\min \{\hat{h}, \tilde{h}\}$ the assumption (A) holds. If not, then there exists a vector $\left(a_{i}\right)_{j=i}^{m} \subset \mathbb{R}^{m}$ such that

$$
\sum_{i=1}^{m}\left(g_{i}, z_{h}\right) a_{i}=0 \quad \forall z_{h} \in Y_{h}
$$

For any $j$ we choose $e_{k} \in Y_{h}$ such that $\left(g_{j}, e_{k}\right) \neq 0$. Hence, $\operatorname{supp}\left(e_{k}\right) \cap \omega_{j} \neq \emptyset$, and $\operatorname{supp}\left(e_{k}\right) \cap \omega_{i}=\emptyset$ holds for every $i \neq j$. Then,

$$
0=\sum_{i=1}^{m}\left(g_{i}, e_{k}\right) a_{i}=\left(g_{j}, e_{k}\right) a_{j},
$$

which implies that $a_{j}=0$. Since $j$ was arbitrary in $\{1, \ldots, m\}$ we arrive at a contradiction.

Theorem 21. Let us assume that $(A)$ holds. Then problem $\left(\mathrm{P}_{\sigma}\right)$ has at least one solution. Moreover, if $\tilde{u}$ is a solution of $\left(\mathrm{P}_{\sigma}\right)$, then $\bar{u}_{\tau}=\left(\Lambda_{\tau} \tilde{u}_{j}\right)_{j=1}^{m}$ is also a solution of $\left(\mathrm{P}_{\sigma}\right)$. In addition, if $f$ is affine with respect to $y$, then $\bar{u}_{\tau}$ is the unique solution belonging to $U_{\tau}^{m}$.

Proof. To establish the existence of a solution $\tilde{u}$ we follow the lines of the proof of Theorem 7. The only concern is the boundedness of the sequence $\left\{a_{k}\right\}_{k=1}^{\infty}$ in $\mathbb{R}^{m}$. For this purpose we consider the difference $z_{\sigma, k}=y_{\sigma, k}-\hat{y}_{\sigma, k}$, where $y_{\sigma, k}$ and $\hat{y}_{\sigma, k}$ are the solutions to (41) corresponding to $u_{k}$ and $\hat{u}_{k}$, respectively. Thus, $z_{\sigma, k}$ is solution of the following system:

$$
\left\{\begin{aligned}
\left(\frac{z_{i, h ; k}-z_{i-1, h ; k}}{\tau_{i}}, z_{h}\right) & +a\left(z_{i, h ; k}, z_{h}\right)+\frac{1}{\tau_{i}} \int_{I_{i}}\left(\partial_{y} f\left(\cdot, t, \xi_{i, h ; k}\right) z_{i, h ; k}, z_{h}\right) d t \\
= & \sum_{j=1}^{m}\left(g_{j}, z_{h}\right) a_{k, j} \quad \forall z_{h} \in Y_{h}, 1 \leq i \leq N_{\tau}, \\
z_{0, h ; k}=0, &
\end{aligned}\right.
$$

where $\xi_{i, h ; k}=\hat{y}_{i, h ; k}+\theta_{i, h ; k}(x, t) z_{i, h ; k}$ with $0 \leq \theta_{i, h ; k}(x, t) \leq 1$.

As in the proof of Theorem 7 we have that $\left\{y_{\sigma, k}\right\}_{k=1}^{\infty}$ and $\left\{\hat{y}_{\sigma, k}\right\}_{k=1}^{\infty}$ are bounded in $L^{2}(Q)$. Since $\mathcal{Y}_{\sigma} \subset L^{\infty}(Q)$ and since $\mathcal{Y}_{\sigma}$ is finite-dimensional, we deduce that $\left\{\hat{y}_{\sigma, k}\right\}_{k=1}^{\infty}$ and $\left\{y_{\sigma, k}\right\}_{k=1}^{\infty}$ are bounded in $L^{\infty}(Q)$ as well. Therefore, the sequences $\left\{\xi_{i, h ; k}\right\}_{k=1}^{\infty}$ are also bounded in $L^{\infty}\left(\Omega \times I_{i}\right)$. Again we argue by contradiction and we assume that $\rho_{k}=\max \left\{\left|a_{k, j}\right|: 1 \leq j \leq m\right\} \rightarrow \infty$ as $k \rightarrow \infty$. Then, we define $\zeta_{\sigma, k}=\frac{1}{\rho_{k}} z_{\sigma, k}$ and $\hat{a}_{k, j}=\frac{a_{k, j}}{\rho_{k}}$. By taking a subsequence we have that $\zeta_{\sigma, k} \rightarrow 0$ in $L^{\infty}(Q)$ and $\hat{a}_{k, j} \rightarrow \hat{a}_{j}, 1 \leq j \leq m$ for some $\left\{\hat{a}_{j}\right\}_{j=1}^{m} \subset \mathbb{R}$. We observe that by definition of $\rho_{k}$ the vector $\hat{a} \neq 0$. Dividing (43) by $\rho_{k}$ we obtain the mentioned subsequence

$$
\left\{\begin{aligned}
\left(\frac{\zeta_{i, h ; k}-\zeta_{i-1, h ; k}}{\tau_{i}}, z_{h}\right) & +a\left(\zeta_{i, h ; k}, z_{h}\right)+\frac{1}{\tau_{i}} \int_{I_{i}}\left(\partial_{y} f\left(\cdot, t, \xi_{i, h ; k}\right) \zeta_{i, h ; k}, z_{h}\right) d t \\
= & \sum_{j=1}^{m}\left(g_{j}, z_{h}\right) \hat{a}_{k, j} \quad \forall z_{h} \in Y_{h}, 1 \leq i \leq N_{\tau} \\
z_{0, h ; k}=0 . &
\end{aligned}\right.
$$

Passing to the limit in this system as $k \rightarrow \infty$ we infer that

$$
\sum_{j=1}^{m}\left(g_{j}, z_{h}\right) \hat{a}_{j}=0 \quad \forall z_{h} \in Y_{h} .
$$


Hence, assumption (A) implies $\hat{a}=0$, which is the desired contradiction. Consequently, the sequence $\left\{a_{k}\right\}_{k=1}^{\infty}$ is bounded, so the existence of a solution $\tilde{u}$ follows by standard arguments.

The fact that $\bar{u}_{\tau}=\left(\Lambda_{\tau} \tilde{u}_{j}\right)_{j=1}^{m}$ is also a solution of $\left(\mathrm{P}_{\sigma}\right)$ is an immediate consequence of Remark 19 and inequality (37). Finally, we prove the uniqueness of a solution in $U_{\tau}^{m}$ if $f$ is affine with respect to the state. First, we observe that both terms in the cost functional are convex in this case. Moreover, the first term is strictly convex on $U_{\tau}^{m}$ provided that the affine mapping $u_{\tau} \rightarrow y_{\sigma}$ is injective. To this end we assume that for some $u_{\tau}=\left(u_{j}\right)_{j=1}^{m} \in U_{\tau}^{m}$, with $u_{j}=\sum_{k=1}^{N_{\tau}} u_{j, k} \chi_{k}$, the associated discrete state $y_{\sigma}$ is identically zero. Then from (41) we have that

$$
\sum_{j=1}^{m}\left(g_{j}, z_{h}\right) u_{j, k}=0 \quad \forall z_{h} \in Y_{h}, \forall 1 \leq k \leq N_{\tau}
$$

Again by assumption (A) we infer that $u_{j}=0$ for every $1 \leq j \leq m$, hence $u_{\tau}=0$.

Remark 22. In the case that $\beta_{j}>0$ for all $1 \leq j \leq m$, condition (A) is not needed to establish the existence of a solution of $\left(\mathrm{P}_{\sigma}\right)$. However, it is still necessary for the uniqueness in the case that $f$ is affine with respect to $y$.

The rest of this section is devoted to the formulation of the first order optimality conditions for the problem $\left(\mathrm{P}_{\sigma}\right)$. Arguing in a similar way as for the continuous problem $(\mathrm{P})$, we separate the smooth and the convex parts of $J_{\sigma}$,

$$
J_{\sigma}(u)=F_{\sigma}(u)+G(u), \quad \text { with } F_{\sigma}(u)=\frac{1}{2}\left\|y_{\sigma}-y_{d}\right\|_{L^{2}\left(Q_{h}\right)}^{2}+\sum_{j=1}^{m} \frac{\beta_{j}}{2}\left(\int_{0}^{T} u_{j}(t) d t\right)^{2},
$$

where $y_{\sigma}$ is related to $u$ by (41). The derivative of $F_{\sigma}$ is expressed by

$$
F_{\sigma}^{\prime}(u) v=\sum_{j=1}^{m} \int_{0}^{T}\left(\int_{\omega_{j}} \varphi_{\sigma}(x, t) g_{j}(x) d x+\beta_{j} \int_{0}^{T} u_{j}(s) d s\right) v_{j}(t) d t,
$$

where $\varphi_{\sigma} \in \mathcal{Y}_{\sigma}$ is the adjoint state associated to $u$, i.e.,

$$
\left\{\begin{aligned}
\left(\frac{\varphi_{k, h}-\varphi_{k+1, h}}{\tau_{k}}, z_{h}\right) & +a\left(\varphi_{k, h}, z_{h}\right)+\frac{1}{\tau_{k}} \int_{I_{k}}\left(\partial_{y} f\left(\cdot, t, y_{k, h}\right) \varphi_{k, h}, z_{h}\right) d t \\
= & \frac{1}{\tau_{k}} \int_{I_{k}}\left(y_{k, h}-y_{d}, z_{h}\right) d t \quad \forall z_{h} \in Y_{h}, k=N_{\tau}, \ldots 1 \\
\varphi_{N_{\tau}+1, h}=0 . &
\end{aligned}\right.
$$

Using this expression for $F_{\sigma}^{\prime}$ and arguing exactly as in the proof of Theorem 9 we obtain the first order optimality conditions for a local solution $\bar{u}_{\tau} \in B V(0, T)^{m}$ of $\left(\mathrm{P}_{\sigma}\right)$. For this purpose we introduce the functions

$$
\bar{\Phi}_{\sigma, j}(t)=\int_{0}^{t} \int_{\omega_{j}} \bar{\varphi}_{\sigma}(x, s) g_{j}(x) d x d s+\beta_{j} t \int_{0}^{T} \bar{u}_{j}(s) d s, \quad 1 \leq j \leq m,
$$

where $\bar{\varphi}_{\sigma} \in \mathcal{Y}_{\sigma}$ is the adjoint state associated to $\bar{u}_{\tau}$. 
THEOREM 23. If $\bar{u}_{\tau}$ is a local solution of $\left(\mathrm{P}_{\sigma}\right)$, then $\bar{\Phi}_{\sigma, j} \in C^{1}[0, T] \cap C_{0}(0, T)$ for $1 \leq j \leq m, \frac{1}{\alpha_{j}} \bar{\Phi}_{\sigma, j} \in \partial g\left(\bar{u}_{\tau, j}^{\prime}\right)$, and they satisfy

$$
\begin{gathered}
\left\|\bar{\Phi}_{\sigma, j}\right\|_{C_{0}(0, T)} \begin{cases}=\alpha_{j} & \text { if } \bar{u}_{\tau, j} \neq 0, \\
\leq \alpha_{j} & \text { if } \bar{u}_{\tau, j}=0,\end{cases} \\
\int_{0}^{T} \bar{\Phi}_{\sigma, j} d \bar{u}_{\tau, j}^{\prime}=\left\|\bar{\Phi}_{\sigma, j}\right\|_{C_{0}(0, T)}\left\|\bar{u}_{\tau, j}^{\prime}\right\|_{\mathcal{M}(0, T)} .
\end{gathered}
$$

In the case where $\bar{u}_{\tau}$ is a local solution of $\left(\mathrm{P}_{\sigma}\right)$ belonging to $U_{\tau}^{m}$ (see Theorem 21), we have the following sparsity result analogous to Corollary 10.

Corollary 24. Let $\bar{u}_{\tau}=\left(\bar{u}_{\tau, j}\right)_{j=1}^{m} \in U_{\tau}^{m}$ be a local solution of $\left(\mathrm{P}_{\sigma}\right)$. Then, for each $j \in\{1, \ldots, m\}$ such that $\bar{u}_{\tau, j}$ is not a constant function on $[0, T]$, we have

$$
\left\{\begin{array}{l}
\bar{u}_{\tau, j}^{++}=\sum_{k \in \mathcal{J}_{\sigma}^{+}}\left(\bar{u}_{j, k+1}-\bar{u}_{j, k}\right) \delta_{t_{k}} \text { with } \mathcal{J}_{\sigma}^{+}=\left\{k \in\left\{1, \ldots, N_{\tau}-1\right\}: \bar{\Phi}_{\sigma, j}\left(t_{k}\right)=+\alpha_{j}\right\}, \\
\bar{u}_{\tau, j}^{\prime-}=\sum_{k \in \mathcal{J}_{\sigma}^{-}}\left(\bar{u}_{j, k+1}-\bar{u}_{j, k}\right) \delta_{t_{k}} \text { with } \mathcal{J}_{\sigma}^{-}=\left\{k \in\left\{1, \ldots, N_{\tau}-1\right\}: \bar{\Phi}_{\sigma, j}\left(t_{k}\right)=-\alpha_{j}\right\},
\end{array}\right.
$$

where $\bar{u}_{\tau, j}^{\prime}=\bar{u}_{\tau, j}^{\prime+}-\bar{u}_{\tau, j}^{\prime-}$ is the Jordan decomposition of the measure $\bar{u}_{\tau, j}^{\prime}$.

Proof. The proof of this result is a consequence of the representation formula for $\bar{u}_{\tau}^{\prime}$ given in (35). In addition, we use $\frac{1}{\alpha_{j}} \bar{\Phi}_{\sigma, j} \in \partial g\left(\bar{u}_{\tau, j}^{\prime}\right)$ along with Proposition 4, and the fact that $\bar{u}_{\tau, j}^{\prime} \neq 0$ by assumption. Finally, we take into account that $\Phi_{\sigma, j}$ is piecewise linear and continuous, and $\Phi_{\sigma, j}(0)=\Phi_{\sigma, j}(T)=0$. Consequently, its maximal and minimal values are attained at the interior grid points $\left\{t_{k}\right\}_{k=1}^{N_{\tau}-1}$.

5. Convergence analysis. The goal of this section is to prove the convergence of solutions of $\left(\mathrm{P}_{\sigma}\right)$ to solutions of $(\mathrm{P})$ as $\sigma \rightarrow 0$. Additionally, we give some error estimates for the difference between the optimal discrete and continuous states.

THEOREM 25. Let us assume that either $f$ is affine with respect to $y$ or $\beta_{j}>0$ for every $1 \leq j \leq m$, and let $\left\{\bar{u}_{\tau}\right\}_{\tau} \subset B V(0, T)^{m}$ be a family of global solutions of problems $\left(\mathrm{P}_{\sigma}\right), \sigma=(h, \tau)$. Then this family is bounded in $B V(0, T)^{m}$. In addition, if $f$ is affine or $n<3$, then any weak $k^{*}$ limit $\bar{u}$ of a subsequence when $\sigma \rightarrow 0$ is a global solution of $(\mathrm{P})$. For such a subsequence we have

$$
\begin{aligned}
& \left\|\bar{u}_{\tau}^{\prime}\right\|_{\mathcal{M}(0, T)^{m}} \rightarrow\left\|\bar{u}^{\prime}\right\|_{\mathcal{M}(0, T)^{m}} \text { and }\left\|\bar{u}-\bar{u}_{\tau}\right\|_{L^{p}(0, T)^{m}} \rightarrow 0 \forall p \in[1,+\infty), \\
& \left\|\bar{y}-\bar{y}_{\sigma}\right\|_{L^{2}(Q)} \rightarrow 0 \text { and } J_{\sigma}\left(\bar{u}_{\tau}\right) \rightarrow J(\bar{u}),
\end{aligned}
$$

where $\bar{y}$ and $\bar{y}_{\sigma}$ are the continuous and discrete states associated to $\bar{u}$ and $\bar{u}_{\tau}$, respectively.

For the proof we will use the following lemma.

LEMmA 26. Let $d_{\sigma} \in L^{2}(Q)$ and take $y_{\sigma} \in \mathcal{Y}_{\sigma}$ to be the solution of

$$
\left\{\begin{array}{l}
\left(\frac{y_{k, h}-y_{k-1, h}}{\tau_{k}}, z_{h}\right)+a\left(y_{k, h}, z_{h}\right)+\frac{1}{\tau_{k}} \int_{I_{k}}\left(f\left(\cdot, t, y_{k, h}\right), z_{h}\right) d t \\
=\frac{1}{\tau_{k}} \int_{I_{k}}\left(d_{\sigma}(t), z_{h}\right) d t \quad \forall z_{h} \in Y_{h}, 1 \leq k \leq N_{\tau}, \\
y_{0, h}=y_{0 h} .
\end{array}\right.
$$


Then, there exists a constant $C_{\Omega}>0$ dependent only on $\Omega$ such that (52)

$$
\left\|y_{\sigma}\right\|_{L^{\infty}\left(0, T ; L^{2}(\Omega)\right)}+\left\|\nabla_{x} y_{\sigma}\right\|_{L^{2}(Q)} \leq C_{\Omega}\left(\left\|d_{\sigma}\right\|_{L^{2}(Q)}+\|f(\cdot, \cdot, 0)\|_{L^{2}(Q)}+\left\|y_{0 h}\right\|_{L^{2}(\Omega)}\right) .
$$

Proof. The proof is standard, except for the nonlinear term. Choosing $z_{h}=y_{k, h}$ in (51), we obtain

$$
\begin{aligned}
& \left(y_{k, h}-y_{k-1, h}, y_{k, h}\right)+\tau_{k} a\left(y_{k, h}, y_{k, h}\right)+\int_{I_{k}}\left(f\left(\cdot, t, y_{k, h}\right)-f(\cdot, t, 0), y_{k, h}\right) d t \\
& =\int_{I_{k}}\left(d_{\sigma}(t)-f(\cdot, t, 0), y_{k, h}\right) d t .
\end{aligned}
$$

Using the monotonicity of $f$ with respect to $y$ we deduce

$$
\left(y_{k, h}-y_{k-1, h}, y_{k, h}\right)+\tau_{k} a\left(y_{k, h}, y_{k, h}\right) \leq \int_{I_{k}}\left(d_{\sigma}(t)-f(\cdot, t, 0), y_{k, h}\right) d t .
$$

The rest of the proof can be completed as in the linear case.

Proof of Theorem 25. Let us set

$$
a_{\tau}=\frac{1}{\tau} \int_{0}^{T} \bar{u}_{\tau} d t \quad \text { and } \quad \hat{u}_{\tau}=\bar{u}_{\tau}-a_{\tau} .
$$

Let $\hat{y}_{\tau}$ be the discrete state associated with $\hat{u}_{\tau}$. The proof is divided into three steps.

Step 1. $\left\{\bar{y}_{\sigma}\right\}_{\sigma}$ and $\left\{\bar{u}_{\tau}\right\}_{\tau}$ are bounded in $L^{2}(Q)$ and $B V(0, T)^{m}$.

From the global optimality of $\bar{u}_{\tau}$ we have that $J_{\sigma}\left(\bar{u}_{\tau}\right) \leq J_{\sigma}(0)$ for every $\sigma$. From Lemma 26, we obtain that the discrete states $y_{\sigma}$ associated to 0 are uniformly bounded in $L^{2}(Q)$. Hence, $\left\{J_{\sigma}(0)\right\}_{\sigma}$ is bounded and, consequently, $\left\{\bar{y}_{\sigma}\right\}_{\sigma}$ and $\left\{\bar{u}_{\tau}^{\prime}\right\}_{\tau}$ are bounded in $L^{2}(Q)$ and $\mathcal{M}(0, T)^{m}$, respectively. According to (2), it is enough to prove the boundedness of $\left\{a_{\tau}\right\}_{\tau}$ in $\mathbb{R}^{m}$ to conclude the boundedness of $\left\{\bar{u}_{\tau}\right\}_{\tau}$ in $B V(0, T)^{m}$. This is obvious if $\beta_{j}>0$ for $1 \leq j \leq m$. Otherwise, by assumption we have that $f=c_{0} y+d_{0}$ with $c_{0} \geq 0, c_{0} \in L^{\infty}(Q)$, and $d_{0} \in L^{\hat{p}}(Q)$.

Let us put $z_{\sigma}=\bar{y}_{\sigma}-\hat{y}_{\sigma}$. Using again (2) we obtain that $\left\{\hat{u}_{\tau}\right\}_{\tau}$ is bounded in $B V(0, T)^{m} \subset L^{2}(Q)^{m}$. Then, Lemma 26 implies the boundedness of $\left\{\hat{y}_{\sigma}\right\}_{\sigma}$ in $L^{2}(Q)$. Thus, we also have the boundedness of $\left\{z_{\sigma}\right\}_{\sigma}$ in $L^{2}(Q)$. Subtracting the discrete equations satisfied by $\bar{y}_{\sigma}$ and $\hat{y}_{\sigma}$ yields

$$
\left\{\begin{aligned}
\left(\frac{z_{k, h}-z_{k-1, h}}{\tau_{k}}, z_{h}\right) & +a\left(z_{k, h}, z_{h}\right)+\frac{1}{\tau_{k}} \int_{I_{k}}\left(c_{0}(\cdot, t) z_{k, h}, z_{h}\right) d t \\
& =\sum_{j=1}^{m}\left(g_{j}, z_{h}\right) a_{\tau, j} \quad \forall z_{h} \in Y_{h}, 1 \leq k \leq N_{\tau}, \\
z_{0, h}=0, &
\end{aligned}\right.
$$

where $a_{\tau}=\left(a_{\tau, j}\right)_{j=1}^{m}$. To argue by contradiction let us assume that

$$
\rho_{\tau}=\max _{1 \leq j \leq m}\left|a_{\tau, j}\right| \rightarrow+\infty \text { as } k \rightarrow \infty .
$$

Then, introducing $\zeta_{\sigma}=\frac{1}{\rho_{\tau}} z_{\sigma}$ and $\bar{a}_{\tau, j}=\frac{a_{\tau, j}}{\rho_{\tau}}$, we deduce from (53)

$$
\left\{\begin{aligned}
\left(\frac{\zeta_{k, h}-\zeta_{k-1, h}}{\tau_{k}}, z_{h}\right)+ & a\left(\zeta_{k, h}, z_{h}\right)+\frac{1}{\tau_{k}} \int_{I_{k}}\left(c_{0}(\cdot, t) \zeta_{k, h}, z_{h}\right) d t \\
& =\sum_{j=1}^{m}\left(g_{j}, z_{h}\right) \bar{a}_{\tau, j} \quad \forall z_{h} \in Y_{h}, 1 \leq k \leq N_{\tau}, \\
\zeta_{0, h}=0 . &
\end{aligned}\right.
$$


By taking a subsequence, that we denote in the same way, we can assume that $\bar{a}_{\tau, j} \rightarrow$ $\bar{a}_{j}$ as $\tau \rightarrow 0$ for every $1 \leq j \leq m$, and $\bar{a}=\left(\bar{a}_{j}\right)_{j=1}^{m} \neq 0$. Let us denote by $\bar{\zeta}_{\sigma}$ the solution of (54) with $\bar{a}_{\tau}$ replaced by $\bar{a}$. From Lemma 26 we deduce that $\left\|\zeta_{\sigma}-\bar{\zeta}_{\sigma}\right\|_{L^{2}(Q)} \rightarrow 0$ as $\sigma \rightarrow 0$. Let $\bar{\zeta} \in H^{2,1}(Q)$ be the solution to

$$
\left\{\begin{aligned}
\frac{\partial \bar{\zeta}}{\partial t}(x, t)-\Delta \bar{\zeta}(x, t)+c_{0} \bar{\zeta} & =\sum_{j=1}^{m} \bar{a}_{j} g_{j} & & \text { in } Q=\Omega \times(0, T), \\
\bar{\zeta}(x, t) & =0 & & \text { on } \Sigma=\Gamma \times(0, T), \\
\bar{\zeta}(x, 0) & =0 & & \text { in } \Omega .
\end{aligned}\right.
$$

From Proposition 17 we infer that $\left\|\bar{\zeta}-\bar{\zeta}_{\sigma}\right\|_{L^{2}(Q)} \rightarrow 0$ as $\sigma \rightarrow 0$. Using the boundedness of $\left\{z_{\sigma}\right\}_{\sigma}$ in $L^{2}(Q)$ and the definition of $\zeta_{\sigma}$ we conclude that $\zeta_{\sigma} \rightarrow 0$ in $L^{2}(Q)$. Hence, $\bar{\zeta}_{\sigma}=\zeta_{\sigma}+\left(\bar{\zeta}_{\sigma}-\zeta_{\sigma}\right) \rightarrow 0$ in $L^{2}(Q)$ as well. This implies that $\bar{\zeta}=0$, and consequently $\sum_{j=1}^{m} \bar{a}_{j} g_{j}=0$. By our assumptions on $\left\{g_{j}\right\}_{j=1}^{m}$ this yields $\bar{a}=0$, which gives the desired contradiction. Therefore, $\left\{\bar{u}_{\tau}\right\}_{\tau}$ is bounded in $B V(0, T)^{m}$.

Let us take a subsequence of $\left\{\bar{u}_{\tau}\right\}_{\tau}$, denoted in the same way, such that $\bar{u}_{\tau} \stackrel{*}{\rightarrow} \bar{u}$ as $\sigma \rightarrow 0$.

Step 2. $\bar{u}$ is a global solution of (P), and (49)-(50) hold.

The compactness of the embedding $B V(0, T) \subset L^{p}(0, T)$ for every $p \in[1,+\infty)$ implies the strong convergence $\bar{u}_{\tau} \rightarrow \bar{u}$ in $L^{p}(0, T)^{m}$. Let us denote by $\bar{y}$ and $\hat{y}_{\sigma}$ the continuous and discrete states corresponding to $\bar{u}$. From Proposition 17 we know that $\hat{y}_{\sigma} \rightarrow \bar{y}$ in $L^{2}(Q)$ as $\sigma \rightarrow 0$. Subtracting the equations satisfied by $\bar{y}_{\sigma}$ and $\hat{y}_{\sigma}$ we obtain for $\zeta_{\sigma}=\bar{y}_{\sigma}-\hat{y}_{\sigma}$

$$
\left\{\begin{array}{l}
\left(\frac{\zeta_{k, h}-\zeta_{k-1, h}}{\tau_{k}}, z_{h}\right)+a\left(\zeta_{k, h}, z_{h}\right)+\frac{1}{\tau_{k}} \int_{I_{k}}\left(\partial_{y} f\left(\cdot, t, \xi_{k, h}\right) \zeta_{k, h}, z_{h}\right) d t \\
=\sum_{j=1}^{m}\left(g_{j}, z_{h}\right) \frac{1}{\tau_{k}} \int_{I_{k}}\left(\bar{u}_{\tau, j}-\bar{u}_{j}\right) d t \quad \forall z_{h} \in Y_{h}, 1 \leq k \leq N_{\tau}, \\
\zeta_{0, h}=0,
\end{array}\right.
$$

where $\xi_{k, h}(x, t)=\hat{y}_{k, h}+\theta_{k, h}(x, t) \zeta_{k, h}$ with $0 \leq \theta_{k, h}(x, t) \leq 1$. In the case of an affine function $f$ we simply have $\partial_{y} f\left(x, t, \xi_{k, h}\right)=c_{0}(x, t)$. Arguing as in Lemma 26 and using that $\partial_{y} f \geq 0$ we get

$$
\left\|\zeta_{\sigma}\right\|_{L^{2}(Q)} \leq C_{\Omega} \max _{1 \leq j \leq m}\left\|g_{j}\right\|_{L^{\infty}(Q)}\left\|\bar{u}-\bar{u}_{\tau}\right\|_{L^{2}(Q)^{m}} \rightarrow 0 \text { as } \sigma \rightarrow 0
$$

Hence, $\bar{y}_{\sigma}=\hat{y}_{\sigma}+\zeta_{\sigma} \rightarrow \bar{y}$ in $L^{2}(Q)$. Now, the following relations hold:

$$
\begin{aligned}
& J(\bar{u}) \leq F(\bar{u})+\liminf _{\sigma \rightarrow 0} G\left(\bar{u}_{\tau}\right) \leq F(\bar{u})+\limsup _{\sigma \rightarrow 0} G\left(\bar{u}_{\tau}\right) \\
& =\lim _{\sigma \rightarrow 0} F_{\sigma}\left(\bar{u}_{\tau}\right)+\limsup _{\sigma \rightarrow 0} G\left(\bar{u}_{\tau}\right)=\limsup _{\sigma \rightarrow 0} J_{\sigma}\left(\bar{u}_{\tau}\right) \\
& \leq \limsup _{\sigma \rightarrow 0} J_{\sigma}(\bar{u})=J(\bar{u})=F(\bar{u})+G(\bar{u}) .
\end{aligned}
$$

As a consequence we have $G(\bar{u})=\lim _{\tau \rightarrow 0} G\left(\bar{u}_{\tau}\right)$. Finally, taking into account that $\left\|\bar{u}_{j}^{\prime}\right\|_{\mathcal{M}(0, T)} \leq \liminf _{\tau \rightarrow 0}\left\|\bar{u}_{\tau, j}^{\prime}\right\|_{\mathcal{M}(0, T)}$ for $1 \leq j \leq m$, we deduce $\left\|\bar{u}_{\tau, j}^{\prime}\right\|_{\mathcal{M}(0, T)} \rightarrow$ $\left\|\bar{u}_{j}^{\prime}\right\|_{\mathcal{M}(0, T)}$ for $1 \leq j \leq m$. This completes the proof.

The next theorem addresses the approximation of local solutions of $(\mathrm{P})$ by local minima of $\left(\mathrm{P}_{\sigma}\right)$. It is in some sense a converse of the previous theorem. 
TheOREm 27. Assume that either $f$ is affine or $n<3$, and let $\bar{u}$ be a strict $L^{p}(0, T)^{m}$-local minimum of $(\mathrm{P})$ with $p \in[1,+\infty)$. Then there exist an $L^{p}(0, T)^{m}$ ball $B_{\rho}(\bar{u})$ such that $J_{\sigma}$ has a global minimum $\bar{u}_{\tau}$ in $\bar{B}_{\rho}(\bar{u}) \cap B V(0, T)^{m}$ for every $\sigma$. The family $\left\{\bar{u}_{\tau}\right\}_{\tau}$ converges to $\bar{u}$ in the sense of (49)-(50). Consequently, there exists $\sigma_{0}$ such that $\bar{u}_{\tau}$ is a local solution of $\left(\mathrm{P}_{\sigma}\right)$ for every $|\sigma| \leq\left|\sigma_{0}\right|$.

Proof. Since $\bar{u}$ is a strict $L^{p}(0, T)^{m}$-local minimum of $(\mathrm{P})$, there exists $\rho>0$ such that

$$
J(\bar{u})<J(u) \quad \forall u \in \bar{B}_{\rho}(\bar{u}) \backslash\{\bar{u}\} .
$$

We consider the problem

$$
\left(\mathrm{P}_{\sigma, \rho}\right) \quad \min \left\{J_{\sigma}(u): u \in B V(0, T)^{m} \cap \bar{B}_{\rho}(\bar{u})\right\} .
$$

The existence of at least one solution $\bar{u}_{\tau}$ for $\left(\mathrm{P}_{\sigma, \rho}\right), \sigma=(h, \tau)$, is obvious. Arguing as in the proof of the previous theorem, we deduce that $\left\{\bar{u}_{\tau}\right\}_{\tau}$ has converging subsequences and any of these limits is a solution of the problem

$$
\min \left\{J(u): u \in B V(0, T)^{m} \cap \bar{B}_{\rho}(\bar{u})\right\} .
$$

Since $\bar{u}$ is the unique solution of $\left(\mathrm{P}_{\rho}\right)$, it follows that the entire family $\left\{\bar{u}_{\tau}\right\}_{\tau}$ converges to $\bar{u}$ in the sense of (49) and (50). Due to the convergence $\left\|\bar{u}-\bar{u}_{\tau}\right\|_{L^{p}(0, T)^{m}} \rightarrow 0$, we deduce the existence of $\sigma_{0}$ such that $\bar{u}_{\tau} \in B_{\rho}(\bar{u})$ for every $|\sigma| \leq\left|\sigma_{0}\right|$, and hence $\bar{u}_{\tau}$ is a local minimum of $\left(\mathrm{P}_{\sigma}\right)$ in the ball $B_{\rho}(\bar{u})$.

The rest of this section is devoted to the analysis of the rate of convergence for the states $\left\|\bar{y}-\bar{y}_{\sigma}\right\|_{L^{2}(Q)}$. Let $\bar{u}$ be a local solution of $(\mathrm{P})$ such that the sufficient second order condition (SSOC) (31) holds. Theorem 14 implies that $\bar{u}$ is a strict strong local solution, and hence it is a strict $L^{p}(0, T)^{m}$-local solution as well. Let $\rho>0$ such that $\bar{u}$ is a global minimum of $J$ in $\bar{B}_{\rho}(\bar{u}) \cap B V(0, T)^{m}$. Let $\left\{\bar{u}_{\tau}\right\}_{\tau}$ be a family of global minima of $J_{\sigma}$ on $\bar{B}_{\rho}(\bar{u}) \cap B V(0, T)^{m}$ converging to $\bar{u}$ in $L^{p}(0, T)^{m}$, for $p>1$. Then we have the following rate of convergence of the associated states.

THEOREM 28. Let us assume that $\bar{u}$ satisfies the (SSOC) and that either $f$ is affine or $n<3$ holds. Then, under the above notations, there exists $C>0$ independent of $\sigma$ such that for all $\sigma$ sufficiently small

$$
\left\|\bar{y}-\bar{y}_{\sigma}\right\|_{L^{2}(Q)} \leq C(\sqrt{\tau}+h) .
$$

Proof. Since $\bar{u}_{\tau} \rightarrow \bar{u}$ in $L^{p}(0, T)^{m}$ with $p>1$, we have that $\left\|y_{\bar{u}_{\tau}}-\bar{y}\right\|_{L^{\infty}(Q)} \rightarrow 0$ as $\sigma \rightarrow 0$, where $y_{\bar{u}_{\tau}}$ is the continuous state corresponding to $\bar{u}_{\tau}$. Let $\epsilon>0$ be as introduced in Corollary 10. Then there exists $\sigma_{\varepsilon}$ such that $\left\|y_{\bar{u}_{\tau}}-\bar{y}\right\|_{L^{\infty}(Q)} \leq \varepsilon$ for every $|\sigma| \leq\left|\sigma_{\varepsilon}\right|$. Utilizing (33) we have

$$
\begin{aligned}
& \frac{\delta}{2}\left\|y_{\bar{u}_{\tau}}-\bar{y}\right\|_{L^{2}(Q)}^{2} \leq J\left(\bar{u}_{\tau}\right)-J(\bar{u}) \\
& =\left[J\left(\bar{u}_{\tau}\right)-\hat{J}_{\sigma}\left(\bar{u}_{\tau}\right)\right]+\left[\hat{J}_{\sigma}\left(\bar{u}_{\tau}\right)-\hat{J}_{\sigma}(\bar{u})\right]+\left[\hat{J}_{\sigma}(\bar{u})-J(\bar{u})\right],
\end{aligned}
$$

where

$$
\hat{J}_{\sigma}(u)=\frac{1}{2}\left\|y_{\sigma}(u)-y_{d}\right\|_{L^{2}(Q)}^{2}+\sum_{j=1}^{M} \frac{\beta_{j}}{2}\left(\int_{0}^{T} u_{j}(t) d t\right)^{2}+G(u) .
$$


Let us estimate these terms. For the first term we use Proposition 17 as follows:

$$
\begin{aligned}
& J\left(\bar{u}_{\tau}\right)-\hat{J}_{\sigma}\left(\bar{u}_{\tau}\right)=\frac{1}{2}\left\|y_{\bar{u}_{\tau}}-y_{d}\right\|_{L^{2}(Q)}^{2}-\frac{1}{2}\left\|\bar{y}_{\sigma}-y_{d}\right\|_{L^{2}\left(Q_{h}\right)}^{2} \\
& \leq C_{1}\left\|y_{\bar{u}_{\tau}}-\bar{y}_{\sigma}\right\|_{L^{2}(Q)} \leq C_{2}\left(\tau+h^{2}\right) .
\end{aligned}
$$

The third term is estimated in the same way, and for the second it is enough to observe

$$
\hat{J}_{\sigma}\left(\bar{u}_{\tau}\right)-\hat{J}_{\sigma}(\bar{u})=J_{\sigma}\left(\bar{u}_{\tau}\right)-J_{\sigma}(\bar{u}) \leq 0,
$$

the last inequality being a consequence of the fact that $J_{\sigma}$ achieves the minimum value in the ball $B_{\rho}(\bar{u}) \cap B V(0, T)^{m}$ at $\bar{u}_{\tau}$. All together this leads to

$$
\left\|y_{\bar{u}_{\tau}}-\bar{y}\right\|_{L^{2}(Q)} \leq C_{3}(\sqrt{\tau}+h)
$$

Finally, we obtain

$$
\left\|\bar{y}-\bar{y}_{\sigma}\right\|_{L^{2}(Q)} \leq\left\|\bar{y}-y_{\bar{u}_{\tau}}\right\|_{L^{2}(Q)}+\left\|y_{\bar{u}_{\tau}}-\bar{y}_{\sigma}\right\|_{L^{2}(Q)} \leq C_{3}(\sqrt{\tau}+h)+C_{4}\left(\tau+h^{2}\right),
$$

where we have used again Proposition 17.

Remark 29. In the case that $f$ is nonlinear and $n=3$, arguing as in the proof of the above theorem and using the inequality of Remark 18, we obtain the estimate

$$
\left\|\bar{y}-\bar{y}_{\sigma}\right\|_{L^{2}(Q)} \leq C\left(\sqrt{\tau}+\left(\log \frac{T}{\tau}\right) h\right) .
$$

Remark 30. Under the assumptions of the above theorem, and supposing that $y_{d} \in L^{2}\left(0, T ; L^{4}(\Omega)\right)$, and using (34) and Proposition 17, we can argue as in $[4$, Theorem 5.1] to deduce that $\left|J(\bar{u})-J_{\sigma}\left(\bar{u}_{\tau}\right)\right| \leq C\left(\tau+h^{2}\right)$. In the case of a nonlinear function $f$ and $n=3$, Remark 18 implies $\left|J(\bar{u})-J_{\sigma}\left(\bar{u}_{\tau}\right)\right| \leq C\left(\tau+\left(\log \frac{T}{\tau}\right)^{2} h^{2}\right)$.

6. Numerical solution. In this section we show how $\left(\mathrm{P}_{\sigma}\right)$ can be solved numerically. We take $f \equiv 0$ and $y_{0} \equiv 0$ in (1), i.e., we consider the case of a linear state equation with zero state at the initial time.

6.1. A fully discrete formulation. Defining $y_{d, \sigma}$ as the $L^{2}\left(Q_{h}\right)$ projection of $y_{d}$ onto $\mathcal{Y}_{\sigma}$, problem $\left(\mathrm{P}_{\sigma}\right)$ can be equivalently expressed as

$$
\min _{u \in B V(0, T)^{m}} \frac{1}{2}\left\|y_{\sigma}-y_{d, \sigma}\right\|_{L^{2}\left(Q_{h}\right)}^{2}+\sum_{j=1}^{m}\left(\alpha_{j}\left\|u_{j}^{\prime}\right\|_{\mathcal{M}(0, T)}+\frac{\beta_{j}}{2}\left(\int_{0}^{T} u_{j}(t) d t\right)^{2}\right) .
$$

Therefore, Theorem 21 guarantees that we can find a solution for $\left(\mathrm{P}_{\sigma}\right)$ by solving

$$
\min _{u_{\tau} \in U_{\tau}^{m}} \frac{1}{2}\left\|y_{\sigma}-y_{d, \sigma}\right\|_{L^{2}\left(Q_{h}\right)}^{2}+\sum_{j=1}^{m}\left(\alpha_{j}\left\|u_{\tau, j}^{\prime}\right\|_{\mathcal{M}(0, T)}+\frac{\beta_{j}}{2}\left(\int_{0}^{T} u_{\tau, j}(t) d t\right)^{2}\right) .
$$

In the following we denote $N_{\rho}=m N_{\tau}$ and $\hat{v}_{\tau}=\left(v_{11}, v_{12}, \ldots, v_{1 N_{\tau}}, v_{21}, \ldots, v_{m N_{\tau}}\right)^{T}$ for every $\hat{v}_{\tau} \in \mathbb{R}^{N_{\rho}}$. Furthermore, let us set

$$
\psi_{j k}: \mathbb{R}^{N_{\rho}} \rightarrow \mathbb{R}, \quad \psi_{j k}\left(\hat{v}_{\tau}\right)=\left|v_{j k}\right|
$$


for $1 \leq j \leq m, 2 \leq k \leq N_{\tau}$, and

$$
\Psi: \mathbb{R}^{N_{\rho}} \rightarrow \mathbb{R}, \quad \Psi\left(\hat{v}_{\tau}\right)=\sum_{j=1}^{m} \alpha_{j} \sum_{k=2}^{N_{\tau}} \psi_{j k}\left(\hat{v}_{\tau}\right) .
$$

Using that every $u_{\tau} \in U_{\tau}^{m}$ can be represented by a coefficient vector $\hat{u}_{\tau} \in \mathbb{R}^{N_{\rho}}$ and defining $\hat{d}_{\tau} \in \mathbb{R}^{N_{\rho}}$ by $d_{j 1}=u_{j 1}$ and $d_{j k}=u_{j k}-u_{j(k-1)}$ for $1 \leq j \leq m$ and $2 \leq k \leq N_{\tau}$, we infer from (35) that $\left(\mathrm{Q}_{\sigma}\right)$ is equivalent to the finite-dimensional optimization problem

$$
\min _{\hat{d}_{\tau} \in \mathbb{R}^{N_{\rho}}} J_{\rho}\left(\hat{d}_{\tau}\right)=\frac{1}{2}\left(S \hat{d}_{\tau}-\hat{y}_{d, \sigma}\right)^{T} M_{\sigma}\left(S \hat{d}_{\tau}-\hat{y}_{d, \sigma}\right)+\frac{1}{2} \hat{d}_{\tau}^{T} Q \hat{d}_{\tau}+\Psi\left(\hat{d}_{\tau}\right),
$$

where $S \in \mathbb{R}^{N_{\sigma} \times N_{\rho}}$ is the discrete control-to-state mapping $d \mapsto y(d)$, and $M_{\sigma} \in$ $\mathbb{R}^{N_{\sigma} \times N_{\sigma}}$ and $Q \in \mathbb{R}^{N_{\rho} \times N_{\rho}}$ are the matrix representations of the quadratic forms appearing in the first and last terms of $\left(\mathrm{Q}_{\sigma}\right)$. The precise form of these matrices can be found in the preprint of this paper.

6.2. Discrete optimality conditions and regularization. Since $J_{\rho}$ is convex, $\hat{d}_{\tau}^{*} \in \mathbb{R}^{N_{\rho}}$ is optimal for $\left(\mathrm{Q}_{\rho}\right)$ if and only if $0 \in \partial J_{\rho}\left(\hat{d}_{\tau}^{*}\right)$. Since both the differentiable and the nondifferentiable part of $J_{\rho}$ are continuous, we obtain from the sum rule that $0 \in \partial J_{\rho}\left(\hat{d}_{\tau}^{*}\right)$ is equivalent to

$$
0 \in S^{T} M_{\sigma}\left(S \hat{d}_{\tau}^{*}-\hat{y}_{d, \sigma}\right)+Q \hat{d}_{\tau}^{*}+\partial \Psi\left(\hat{d}_{\tau}^{*}\right)
$$

where we have used that $M_{\sigma}$ and $Q$ are symmetric. Thus, $\hat{d}_{\tau}^{*}$ is optimal for $\left(\mathrm{Q}_{\rho}\right)$ if and only if there exists $\hat{\lambda}_{\tau}^{*} \in \mathbb{R}^{N_{\rho}}$ such that

$$
S^{T} M_{\sigma}\left(S \hat{d}_{\tau}^{*}-\hat{y}_{d, \sigma}\right)+Q \hat{d}_{\tau}^{*}-\hat{\lambda}_{\tau}^{*}=0 \quad \text { and } \quad-\hat{\lambda}_{\tau}^{*} \in \partial \Psi\left(\hat{d}_{\tau}^{*}\right) .
$$

The sum rule and the chain rule (cf. [11, Chapter I, Proposition 5.7]) yield that $\partial \Psi\left(\hat{d}_{\tau}^{*}\right) \subset \mathbb{R}^{N_{\rho}}$ is given by

$\partial \Psi\left(\hat{d}_{\tau}^{*}\right)=\{0\} \times \alpha_{1} \partial \psi\left(d_{12}^{*}\right) \times \cdots \times \alpha_{1} \partial \psi\left(d_{1 N_{\tau}}^{*}\right) \times\{0\} \times \alpha_{2} \partial \psi\left(d_{22}^{*}\right) \times \cdots \times \alpha_{m} \partial \psi\left(d_{m N_{\tau}}^{*}\right)$,

where $\psi: \mathbb{R} \rightarrow \mathbb{R}$ denotes $\psi(x)=|x|$. We recognize in $S^{T} M_{\sigma}\left(S \hat{d}_{\tau}^{*}-\hat{y}_{d, \sigma}\right)+Q \hat{d}_{\tau}^{*}$ the discrete version of $\left(\bar{\Phi}_{j}\right)_{j=1}^{m}$ (cf. (20)), which indicates that first-discretize-thenoptimize and first-optimize-then-discretize coincide. To enable the use of semismooth Newton methods we proceed in two steps. The first step is to apply a regularization to $\left(\mathrm{Q}_{\rho}\right)$. More precisely, instead of $\left(\mathrm{Q}_{\rho}\right)$ we consider for $\gamma>0$ the problem

$$
\left(\mathrm{Q}_{\rho, \gamma}\right) \quad \min _{\hat{d}_{\tau} \in \mathbb{R}^{N_{\rho}}} \frac{1}{2}\left(S \hat{d}_{\tau}-\hat{y}_{d, \sigma}\right)^{T} M_{\sigma}\left(S \hat{d}_{\tau}-\hat{y}_{d, \sigma}\right)+\frac{1}{2} \hat{d}_{\tau}^{T} Q \hat{d}_{\tau}+\Psi_{\gamma}\left(\hat{d}_{\tau}\right),
$$

where $\Psi_{\gamma}$ is defined by

$$
\Psi_{\gamma}: \mathbb{R}^{N_{\rho}} \rightarrow \mathbb{R}, \quad \Psi_{\gamma}\left(\hat{d}_{\tau}\right)=\sum_{j=1}^{m} \alpha_{j} \sum_{k=2}^{N_{\tau}} \psi_{j k}^{\gamma}\left(\hat{d}_{\tau}\right)
$$

with

$$
\psi_{j k}^{\gamma}: \mathbb{R}^{N_{\rho}} \rightarrow \mathbb{R}, \quad \psi_{j k}^{\gamma}\left(\hat{d}_{\tau}\right)=\left|d_{j k}\right|+\frac{\gamma}{2 \tau_{k}}\left|d_{j k}\right|^{2}
$$


for $1 \leq j \leq m$ and $2 \leq k \leq N_{\tau}$. We notice that $\left(\mathrm{Q}_{\rho, \gamma}\right)$ can be interpreted as the discrete counterpart of $\min _{u \in H^{1}(0, T)^{m}} \frac{1}{2}\left\|y_{u}-y_{d}\right\|_{L^{2}(Q)}^{2}+\sum_{j=1}^{m}\left(\alpha_{j}\left(\left\|u_{j}^{\prime}\right\|_{L^{1}(0, T)}+\frac{\gamma}{2}\left\|u_{j}^{\prime}\right\|_{L^{2}(0, T)}^{2}\right)+\frac{\beta_{j}}{2}\left(\int_{0}^{T} u_{j}(t) d t\right)^{2}\right)$.

Since there holds $\left\|u_{j}^{\prime}\right\|_{L^{1}(0, T)}=\left\|u_{j}^{\prime}\right\|_{\mathcal{M}(0, T)}$ for this problem due to $u_{j}^{\prime} \in L^{1}(0, T)$, this problem can be regarded as a regularized version of $(\mathrm{P})$.

Arguing as above we obtain that $\left(\mathrm{Q}_{\rho, \gamma}\right)$ has the optimality conditions (60), but with $\partial \Psi$ replaced by $\partial \Psi_{\gamma}$. In addition, $\partial \Psi_{\gamma}$ has the same structure as $\partial \Psi$, but with $\partial \psi$ in the component $j k$ replaced by $\partial \psi_{\gamma}^{k}$, where $\psi_{\gamma}^{k}: \mathbb{R} \rightarrow \mathbb{R}$ denotes $\psi_{\gamma}^{k}(x)=|x|+\frac{\gamma}{2 \tau_{k}}|x|^{2}$. In the second step, we rewrite $-\hat{\lambda}_{\tau}^{*} \in \partial \Psi_{\gamma}\left(\hat{d}_{\tau}^{*}\right)$ componentwise as $-\lambda_{j k}^{*} / \alpha_{j} \in \partial \psi_{\gamma}^{k}\left(d_{j k}^{*}\right)$ for $1 \leq j \leq m$ and $2 \leq k \leq N_{\tau}$, and replace each of these conditions equivalently by $d_{j k}^{*} \in \partial\left(\psi_{\gamma}^{k}\right)^{*}\left(-\lambda_{j k}^{*} / \alpha_{j}\right)$, where $\left(\psi_{\gamma}^{k}\right)^{*}$ denotes the conjugate function of $\psi_{\gamma}^{k}$, given by $\left(\psi_{\gamma}^{k}\right)^{*}(y)=\sup _{x \in \mathbb{R}}\left(y x-\psi_{\gamma}^{k}(x)\right)$; cf. [11, Chapter I, Corollary 5.2]. Note that for $k=1$ we keep the conditions $\lambda_{j k}^{*}=0$. A straightforward computation reveals that $\left(\psi_{\gamma}^{k}\right)^{*}$ is the continuously differentiable function

$$
\left(\psi_{\gamma}^{k}\right)^{*}(y)=\frac{\tau_{k}}{2 \gamma} \cdot \begin{cases}(y+1)^{2} & \text { if } y \leq-1 \\ 0 & \text { if }-1<y<1 \\ (y-1)^{2} & \text { if } y \geq 1\end{cases}
$$

Therefore, the optimality conditions of $\left(\mathrm{Q}_{\rho, \gamma}\right)$ can be recast as

$S^{T} M_{\sigma}\left(S \hat{d}_{\tau}^{*}-\hat{y}_{d, \sigma}\right)+Q \hat{d}_{\tau}^{*}-\hat{\lambda}_{\tau}^{*}=0, \quad-\frac{\lambda_{j 1}^{*}}{\alpha_{j}}=0, \quad$ and $\quad d_{j k}^{*}=\left(\left(\psi_{\gamma}^{k}\right)^{*}\right)^{\prime}\left(-\frac{\lambda_{j k}^{*}}{\alpha_{j}}\right)$

for $1 \leq j \leq m$ and $2 \leq k \leq N_{\tau}$. This reads $F_{\gamma}\left(\hat{d}_{\tau}^{*}, \hat{\lambda}_{\tau}^{*}\right)=0$ if we let

$$
F_{\gamma}: \mathbb{R}^{N_{\rho}} \times \mathbb{R}^{N_{\rho}} \rightarrow \mathbb{R}^{N_{\rho}} \times \mathbb{R}^{N_{\rho}}, \quad F_{\gamma}\left(\hat{d}_{\tau}, \hat{\lambda}_{\tau}\right)=\left(\begin{array}{c}
S^{T} M_{\sigma}\left(S \hat{d}_{\tau}-\hat{y}_{d, \sigma}\right)+Q \hat{d}_{\tau}+\hat{\lambda}_{\tau}^{\alpha} \\
F_{\gamma, 1}\left(\hat{d}_{\tau}, \hat{\lambda}_{\tau}\right) \\
\vdots \\
F_{\gamma, m}\left(\hat{d}_{\tau}, \hat{\lambda}_{\tau}\right)
\end{array}\right)
$$

where we have employed the definition $\left(\hat{\lambda}_{\tau}^{\alpha}\right)_{j k}=\alpha_{j} \lambda_{j k}$ for $1 \leq j \leq m$ and $1 \leq k \leq N_{\tau}$, and used for $1 \leq j \leq m$ the mappings $F_{\gamma, j}: \mathbb{R}^{N_{\rho}} \times \mathbb{R}^{N_{\rho}} \rightarrow \mathbb{R}^{N_{\tau}}$ given by

$$
F_{\gamma, j}\left(\hat{d}_{\tau}, \hat{\lambda}_{\tau}\right)=\gamma\left(\begin{array}{c}
0 \\
\frac{d_{j 2}}{\tau_{2}} \\
\vdots \\
\frac{d_{j N_{\tau}}}{\tau_{N_{\tau}}}
\end{array}\right)-\left(\begin{array}{c}
\lambda_{j 1} \\
\left(\lambda_{j 2}+1\right)^{-}+\left(\lambda_{j 2}-1\right)^{+} \\
\vdots \\
\left(\lambda_{j N_{\tau}}+1\right)^{-}+\left(\lambda_{j N_{\tau}}-1\right)^{+}
\end{array}\right)
$$

Since $F_{\gamma}$ is semismooth, we can apply a semismooth Newton method to solve $F_{\gamma}=0$. For later reference we note that the Newton step $\hat{s}_{\tau}=\left(\hat{s}_{d}, \hat{s}_{\lambda}\right)$ at $\left(\hat{d}_{\tau}, \hat{\lambda}_{\tau}\right)$ solves (61)

$$
F_{\gamma}^{\prime}\left(\hat{d}_{\tau}, \hat{\lambda}_{\tau}\right) \hat{s}_{\tau}=-F_{\gamma}\left(\hat{d}_{\tau}, \hat{\lambda}_{\tau}\right) \quad \text { with } \quad F_{\gamma}^{\prime}\left(\hat{d}_{\tau}, \hat{\lambda}_{\tau}\right)=\left(\begin{array}{cc}
S^{T} M_{\sigma} S+Q & \operatorname{diag}(\hat{\alpha}) \\
\gamma \operatorname{diag}(w) & -\operatorname{diag}\left(e\left(\hat{\lambda}_{\tau}\right)\right)
\end{array}\right) .
$$


Here, we have used $\hat{\alpha}, w, e\left(\hat{\lambda}_{\tau}\right) \in \mathbb{R}^{N_{\rho}}$, defined componentwise by $(\hat{\alpha})_{j k}=\alpha_{j}$,

$$
(w)_{j k}=\left\{\begin{array}{ll}
0 & \text { if } k=1, \\
\frac{1}{\tau_{k}} & \text { if } k \neq 1,
\end{array} \quad \text { and } \quad\left(e\left(\hat{\lambda}_{\tau}\right)\right)_{j k}= \begin{cases}1 & \text { if } k=1 \\
0 & \text { if } k \neq 1 \text { and } \lambda_{j k} \in(-1,1), \\
1 & \text { if } k \neq 1 \text { and } \lambda_{j k} \notin(-1,1)\end{cases}\right.
$$

for $1 \leq j \leq m$ and $1 \leq k \leq N_{\tau}$.

6.3. Path-following algorithm. Since we have approximated $\left(\mathrm{Q}_{\rho}\right)$ by $\left(\mathrm{Q}_{\rho, \gamma}\right)$, we consider a path-following algorithm that drives $\gamma$ to zero. It is called Algorithm BV. In this algorithm we use the definition $\|\hat{v}\|_{L^{2}(0, T)^{2 m}}=\sum_{j=1}^{2 m}\left(\sum_{k=1}^{N_{\tau}} \tau_{k} v_{j k}^{2}\right)^{\frac{1}{2}}$ for $\hat{v}=$ $\left(v_{11}, \ldots, v_{1 N_{\tau}}, v_{21}, \ldots, v_{(2 m) N_{\tau}}\right)^{T} \in \mathbb{R}^{N_{\rho}} \times \mathbb{R}^{N_{\rho}}$.

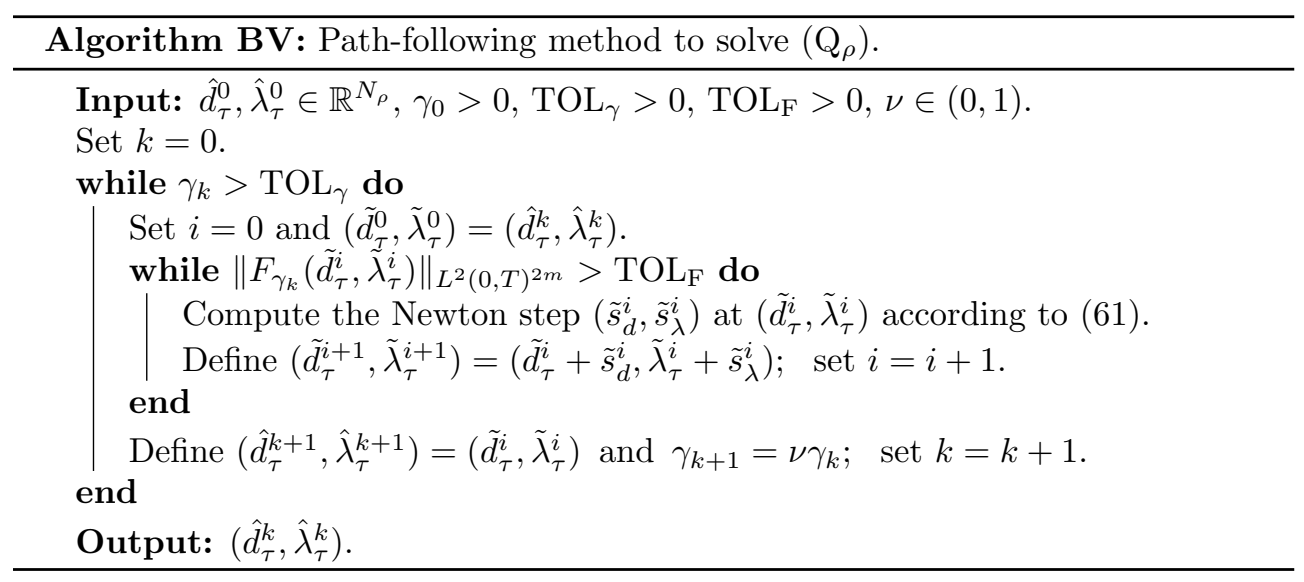

Several variants of this algorithm are conceivable. For instance, a damping strategy could be included, TOL $\mathrm{F}$ could depend on $\gamma_{k}$, and $\nu$ could vary with $k$.

Regarding the convergence behavior of Algorithm BV we point out that the semismooth Newton method for $F_{\gamma}$ converges locally at a q-superlinear rate to the unique solution of $\left(\mathrm{Q}_{\rho, \gamma}\right)$. To prove this it suffices to establish that $\left(\hat{d}_{\tau}, \hat{\lambda}_{\tau}\right) \mapsto\left\|F_{\gamma}^{\prime}\left(\hat{d}_{\tau}, \hat{\lambda}_{\tau}\right)^{-1}\right\|$ is bounded; cf. [24, Proposition 2.12]. Using (61) it can be shown that $F_{\gamma}^{\prime}$ is invertible and that $\left\{F_{\gamma}^{\prime}\left(\hat{d}_{\tau}, \hat{\lambda}_{\tau}\right):\left(\hat{d}_{\tau}, \hat{\lambda}_{\tau}\right) \in \mathbb{R}^{N_{\rho}} \times \mathbb{R}^{N_{\rho}}\right\} \subset \mathbb{R}^{2 N_{\rho} \times 2 N_{\rho}}$ contains only a finite number of elements. This implies, in particular, the asserted boundedness.

7. Numerical examples. We illustrate our findings by three examples. Our main goal is to exemplify the structure of optimal controls for $(\mathrm{P})$. Throughout, we treat the case where $f \equiv 0, \beta_{j}=0$ for all $j$, and $y_{0} \equiv 0$. In particular, $(\mathrm{P})$ is convex and Theorem 7 yields the existence of a unique and global optimal solution.

In all examples we consider controls defined on $(0, T)=(0,2)$ and employ uniformly spaced temporal and spatial grids. We found $\gamma_{0}=1, \mathrm{TOL}_{\mathrm{F}}=10^{-12}$, $\mathrm{TOL}_{\gamma}=10^{-14}$, as well as $\nu=0.1$ (for the majority of examples), and $\nu=0.5$ (for some examples) to be reliable choices in Algorithm BV. We use $\hat{d}_{\tau}^{0}=0$ and take $\hat{\lambda}_{\tau}^{0}$ such that $\left(\hat{d}_{\tau}^{0}, \hat{\lambda}_{\tau}^{0}\right)$ satisfies the condition $S^{T} M_{\sigma}\left(S \hat{d}_{\tau}-\hat{y}_{d, \sigma}\right)+\hat{\lambda}_{\tau}^{\alpha}=0$ in the optimality system $F_{\gamma}=0$. When $\gamma_{k}$ reaches $\mathrm{TOL}_{\gamma}$, the inner while loop in Algorithm BV is executed until $\left|\left\|F_{\gamma_{k}}\left(\tilde{d}_{\tau}^{i}, \tilde{\lambda}_{\tau}^{i}\right)\right\|_{L^{2}(0, T)^{2 m}}-\left\|F_{\gamma_{k}}\left(\tilde{d}_{\tau}^{i-1}, \tilde{\lambda}_{\tau}^{i-1}\right)\right\|_{L^{2}(0, T)^{2 m}}\right| \leq \mathrm{TOL}_{\mathrm{F}}$ and $\left\|F_{\gamma_{k}}\left(\tilde{d}_{\tau}^{i}, \tilde{\lambda}_{\tau}^{i}\right)\right\|_{L^{2}(0, T)^{2 m}} \leq \mathrm{TOL}_{\mathrm{F}}$ are satisfied for three consecutive $i$. We use GMRES to solve the nonsymmetric linear system (61) to a relative accuracy of $10^{-12}$. 
Due to the presence of $S$ and $S^{T}$ in (61), each iteration of GMRES requires to solve two PDEs. These PDE solves are performed to a relative accuracy of $10^{-12}$ using preconditioned GMRES.

7.1. Example 1: One control and one spatial dimension. We start with an example in which $m=1, \Omega=(-1,1)$, and $\omega=(0,1)$. The remaining specifications are made such that an exact analytic solution $\bar{u}$ of $(\mathrm{P})$ is known. The optimal control $\bar{u}$ exhibits $l \in \mathbb{N}$ jumps and it is constant apart from these jumps. Consider

$$
\min _{u \in B V(0, T)} \frac{1}{2}\left\|y_{u}-y_{d}\right\|_{L^{2}(Q)}^{2}+\bar{\alpha}\left\|u^{\prime}\right\|_{\mathcal{M}(0, T)},
$$

where $y_{u}$ is the solution to the parabolic state equation

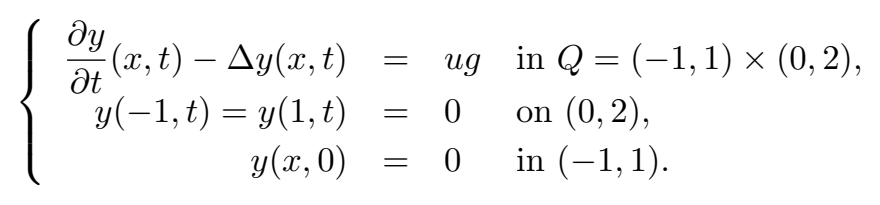

We take $g \equiv 1$ in $\omega$ and $g \equiv 0$ elsewhere, i.e., $g=\chi_{\omega}$. Let $\kappa>0, l \in \mathbb{N}$, and $c_{k} \geq 0$ for $1 \leq k \leq l$. Define

$$
\bar{\alpha}=\frac{4 \kappa}{l \pi^{2}}, \quad \bar{\varphi}(x, t)=\kappa \sin (l \pi t) \cos \left(\frac{\pi}{2} x\right), \quad \bar{u}= \begin{cases}0 & \text { if } t<\frac{1}{l}, \\ c_{1} & \text { if } \frac{1}{l}<t<\frac{3}{l}, \\ \vdots & \vdots \\ \sum_{k=1}^{l} c_{k} & \text { if } \frac{2 l-1}{l}<t<2 .\end{cases}
$$

In particular, this implies $\bar{u}^{\prime}=\sum_{k=1}^{l} c_{k} \delta_{\frac{2 k-1}{l}}$ and $\left\|\bar{u}^{\prime}\right\|_{\mathcal{M}(0, T)}=\sum_{k=1}^{l} c_{k}$. Denoting by $L$ the differential operator $\frac{\partial}{\partial t}-\Delta$ we set $y_{d}=\bar{y}-L^{*} \bar{\varphi}$, where $\bar{y}=y_{\bar{u}}$. To conclude that $\bar{u}$ is the optimal solution of the above optimization problem, we check if $\bar{u}$ satisfies the necessary optimality conditions of Theorem 9 . Since we are dealing with a convex problem, this is already sufficient for global optimality. Alternatively, the optimality of $\bar{u}$ can be established using the conditions from Theorem 14, in particular the condition (SSOC). Considering the first order conditions from Theorem 9, we first note that the adjoint equation $L^{*} \varphi_{\bar{u}}=y_{\bar{u}}-y_{d}$ together with boundary conditions is satisfied by construction. Second, we confirm that

$$
\bar{\Phi}(t)=\int_{0}^{t} \int_{\omega} \bar{\varphi}(x, s) d x d s=\frac{2 \kappa}{l \pi^{2}}(1-\cos (l \pi t))=\frac{\bar{\alpha}}{2}(1-\cos (l \pi t)),
$$

which demonstrates $\bar{\Phi} \in C^{1}[0,2] \cap C_{0}(0,2)$ and $\bar{\Phi}(t) \in[0, \bar{\alpha}]$ for all $t \in[0,2]$, with $\bar{\Phi}(t)=\bar{\alpha}$ exactly for $t=\frac{2 k-1}{l}$ with $1 \leq k \leq l$. Hence, we have $\|\bar{\Phi}\|_{C_{0}(0, T)}=\bar{\alpha}$ and

$$
\int_{0}^{T} \bar{\Phi} d \bar{u}^{\prime}=\sum_{k=1}^{l} c_{k} \bar{\Phi}\left(\frac{2 k-1}{l}\right)=\bar{\alpha} \sum_{k=1}^{l} c_{k}=\|\bar{\Phi}\|_{C_{0}(0, T)}\left\|\bar{u}^{\prime}\right\|_{\mathcal{M}(0, T)},
$$

which establishes (21) and (22). Thus, $\bar{u}$ is optimal. In view of Corollary 10 we note

$$
\left\{\begin{array}{l}
\operatorname{supp}\left(\bar{u}^{\prime+}\right)=\operatorname{supp}\left(\overline{\mathrm{u}}^{\prime}\right) \subset\{\mathrm{t} \in[0, \mathrm{~T}]: \bar{\Phi}(\mathrm{t})=\bar{\alpha}\} \\
\operatorname{supp}\left(\bar{u}^{-}\right)=\{t \in[0, T]: \bar{\Phi}(t)=-\bar{\alpha}\}=\emptyset
\end{array}\right.
$$


where the inclusion is an equality if and only if all $c_{k}$ are positive. Since we have

$$
J(\bar{u})=\frac{1}{2}\left\|y_{\bar{u}}-y_{d}\right\|_{L^{2}(Q)}^{2}+\bar{\alpha}\left\|\bar{u}^{\prime}\right\|_{\mathcal{M}(0, T)}=\frac{1}{2}\left\|L^{*} \bar{\varphi}\right\|_{L^{2}(Q)}^{2}+\frac{4 \kappa}{l \pi^{2}} \sum_{k=1}^{l} c_{k}
$$

and we easily compute $\left\|L^{*} \bar{\varphi}\right\|_{L^{2}(Q)}^{2}=\kappa^{2} \pi^{2}\left(l^{2}+\frac{\pi^{2}}{16}\right)$, the optimal value is given by

$$
J(\bar{u})=\frac{\kappa^{2} \pi^{2}}{2}\left(l^{2}+\frac{\pi^{2}}{16}\right)+\frac{4 \kappa}{l \pi^{2}} \sum_{k=1}^{l} c_{k} .
$$

For the numerical experiments we choose $l=5, \kappa=0.01, c_{1}=c_{3}=c_{5}=2$, and $c_{2}=$ $c_{4}=1$, which yields $\bar{\alpha}=1 /\left(125 \pi^{2}\right) \approx 8.1 \cdot 10^{-4}$ and $J(\bar{u}) \approx 1.9 \cdot 10^{-2}$. Furthermore, it implies that $\bar{u}$ exhibits five jumps, which occur exactly at those $t$ where $\bar{\Phi}(t)=\bar{\alpha}$. Unless indicated otherwise we employ $N_{t}=2560$ and $N_{h}=255$, which corresponds to $\tau=1 / 1280$ and $h=1 / 128$. Application of Algorithm BV yields $\bar{y}_{\sigma}, \bar{u}_{\tau}$, and the optimal dual variable $\bar{\lambda}_{\tau}$, which can be interpreted as discretization of $\bar{\lambda}=\frac{1}{\bar{\alpha}} \bar{\Phi}=$ $\frac{1}{2}(1-\cos (5 \pi t))$. These quantities - more precisely, linear interpolations of them - are depicted together with $y_{d, \sigma}$ in Figure 1 . We observe that $\bar{u}_{\tau}$ and $\bar{\lambda}_{\tau}$ resemble closely their continuous counterparts $\bar{u}$ and $\bar{\lambda}$. In particular, $\bar{u}_{\tau}$ clearly displays the five distinct jumps of $\bar{u}$.

To assess the discretization errors we apply Algorithm BV on different grids, where each grid satisfies $N_{\tau}=10\left(\left(N_{h}+1\right) / 16\right)^{2}$. We use $N_{h}+1=2^{j}$ with $4 \leq j \leq$ 8. The resulting errors $\left\|\bar{y}-\bar{y}_{\sigma}\right\|_{L^{2}(Q)}$ and $\left|J(\bar{u})-J_{\sigma}\left(\bar{u}_{\tau}\right)\right|$ are plotted in Figure 2 . Moreover, this figure shows the error $\left\|\bar{y}-y_{\sigma}(\bar{u})\right\|_{L^{2}(Q)}$. To evaluate $\left\|\bar{y}-\bar{y}_{\sigma}\right\|_{L^{2}(Q)}$ and $\left\|\bar{y}-y_{\sigma}(\bar{u})\right\|_{L^{2}(Q)}$ we require $\bar{y}$. Since $\bar{y}$ is not known explicitly, we compute $y_{\sigma}(\bar{u})$ on a very fine grid and use it as a replacement. The grid for the computation of $y_{\sigma}(\bar{u})$ is described by $N_{h}+1=2^{9}$ and, as before, $N_{\tau}=10\left(\left(N_{h}+1\right) / 16\right)^{2}$, which gives $\tau=10240$ and $N_{h}=511$. Let us point out that the large number of time steps is a consequence of the choice $\tau=\tau(h)=\mathcal{O}\left(h^{2}\right)$ that we make since the error estimates in Theorem 28 and Remark 30 predict convergence order $\mathcal{O}(\sqrt{\tau}+h)$, respectively, $\mathcal{O}\left(\tau+h^{2}\right)$. For the error $\left\|\bar{y}-\bar{y}_{\sigma}\right\|_{L^{2}(Q)}$ we observe quadratic convergence in Figure 2, which is better than the result from Theorem 28. This agrees to some extent with previous contributions on optimal control with measures (cf. [3, 4, 15, 18]), where it is also observed that this error decays faster than linear. The error $\left\|\bar{y}-y_{\sigma}(\bar{u})\right\|_{L^{2}(Q)}$ converges quadratically, which is in accordance with Proposition 17. The optimal objective value appears to converge at a cubic rate. This is faster than we would expect from Remark 30.

Next, we investigate the influence of $\alpha$ on solutions of $(\mathrm{P})$. For this purpose we continue to work with $l=5, \kappa=0.01, c_{1}=c_{3}=c_{5}=2$, and $c_{2}=c_{4}=1$. In particular, we keep the corresponding $y_{d}$. However, instead of $\bar{\alpha}=1 /\left(125 \pi^{2}\right)$ we use

$$
\alpha_{\theta}=\theta \bar{\alpha} \quad \text { with } \quad \theta \in\left[10^{-3}, 10^{2}\right]
$$

in the objective. We stress that for $\theta \neq 1$ we do not know the exact solution of $(\mathrm{P})$. Employing $L^{*} \bar{\varphi}=\kappa\left(\frac{\pi^{2}}{4} \sin (l \pi t) \cos \left(\frac{\pi}{2} x\right)-l \pi \cos (l \pi t) \cos \left(\frac{\pi}{2} x\right)\right)$ it follows from the definition that $y_{d}$ does not satisfy the initial condition $y(x, 0) \equiv 0$ of the state equation. This implies $\bar{y} \neq y_{d}$ regardless of the value of $\theta$. Figures 3 , 4 , and 5 show $\bar{y}_{\sigma}=\bar{y}_{\sigma}^{\theta}$, $\bar{u}_{\tau}=\bar{u}_{\tau}^{\theta}$, and $\bar{\lambda}_{\tau}=\bar{\lambda}_{\tau}^{\theta}$ for different values of $\theta$. We observe that $\bar{u}_{\tau}^{\theta}$ is constant for $\theta=100$. Although not depicted, this is also true for every $\theta>100$ that we tested. Hence, in accordance with Remark 11 the optimal control is constant for sufficiently 

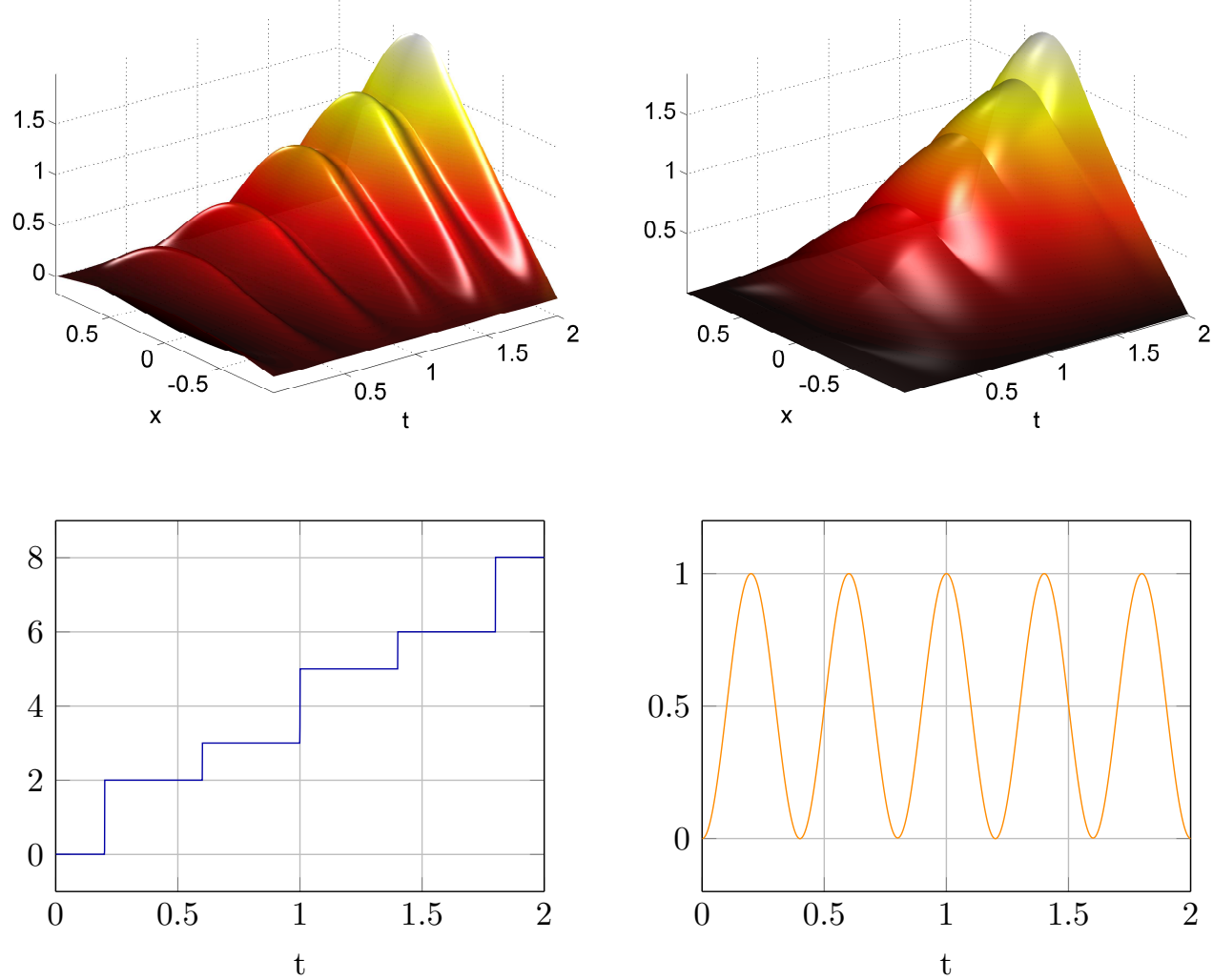

FiG. 1. Example 1: $y_{d, \sigma}$ and $\bar{y}_{\sigma}$ (top left and right), $\bar{u}_{\tau}$ and $\bar{\lambda}_{\tau}$ (bottom left and right).

large values of $\alpha$. As $\theta$ decreases, the number of jumps of $\bar{u}_{\tau}^{\theta}$ increases. For $\theta<1$ jumps with negative height occur. Approximately around $\theta=0.1$ the measures of $\operatorname{supp}\left(\left(\bar{u}_{\tau}^{\theta}\right)^{\prime}\right)$ and $\left\{t \in(0, T): \bar{\lambda}_{\tau}^{\theta}(t)= \pm 1\right\}$ become positive. As $\theta$ decreases further, these measures increase further.

To draw a comparison between $(\mathrm{P})$ and the classical $L^{2}$-regularized tracking problem, we now replace $\alpha_{\theta}\left\|u^{\prime}\right\|_{\mathcal{M}(0, T)}$ in the objective by $\frac{\alpha_{\theta}}{2}\|u\|_{L^{2}(0, T)}^{2}$. The discretization of $\frac{\alpha_{\theta}}{2}\|u\|_{L^{2}(0, T)}^{2}$ is given by $\frac{\alpha_{\theta}}{2} \hat{d}_{\tau}^{T} \tilde{Q}^{T} \tilde{Q} \hat{d}_{\tau}$ with $\tilde{Q} \in \mathbb{R}^{N_{\tau} \times N_{\tau}}$. The precise form of $\tilde{Q}$ can be found in the preprint of this paper. Figure 6 depicts the optimal controls $\bar{u}_{\tau, L^{2}}^{\theta}$ that we obtain for $\alpha_{\theta}=\theta \bar{\alpha}$ and various values of $\theta$. Figure 7 shows the corresponding tracking errors $\frac{1}{2}\left\|\bar{y}_{\sigma, L^{2}}^{\theta}-y_{d, \sigma}\right\|_{L^{2}\left(Q_{h}\right)}^{2}$ as well as the tracking errors for (P). It also displays the norms of the controls as they appear in the objective. The missing data point for the norm of the $\mathrm{BV}$-control at $\theta=100$ results from the fact that the corresponding control is constant, hence its BV-seminorm equals zero. We observe that the tracking errors for both control problems have a similar order of magnitude. From a practical point of view, however, the controls of $(\mathrm{P})$ have a simpler structure. We note, in particular, that for $\theta \approx 5$ the tracking errors are approximately equal for the $L^{2}$ and $\mathrm{BV}$-seminorm cases. The BV-control, however, is cheaper and also reproduces four jumps, whereas the $L^{2}$-control has a complicated structure. 

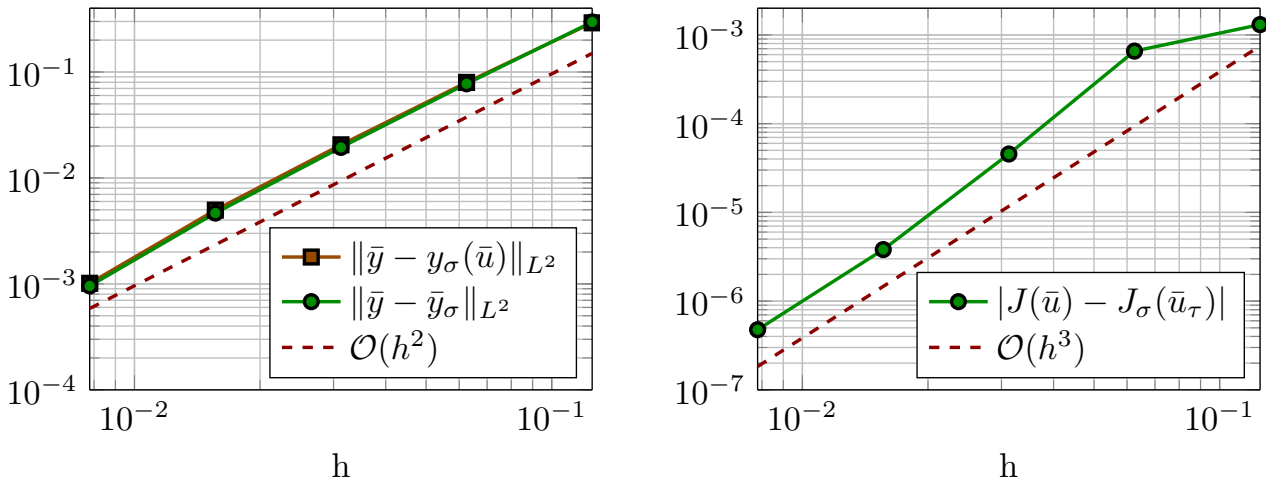

FIG. 2. Example 1: Discretization errors for optimal state and optimal objective value.

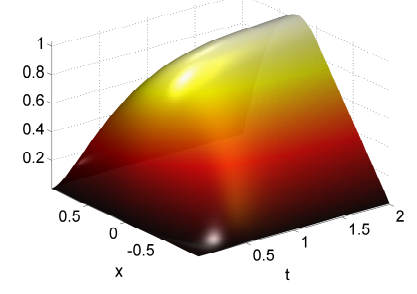

$\theta=100$

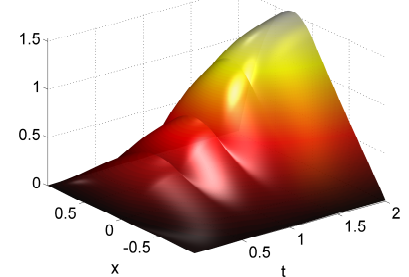

$\theta=10$

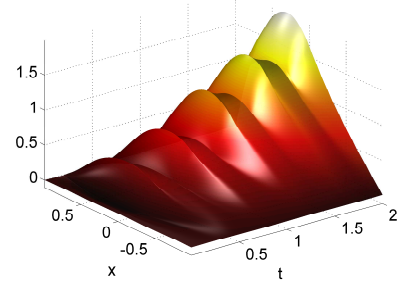

$\theta=0.1$

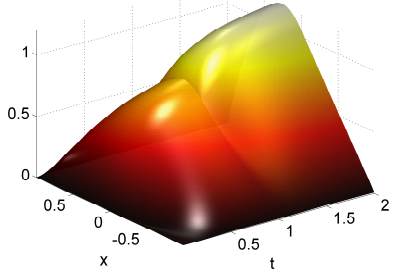

$\theta=50$

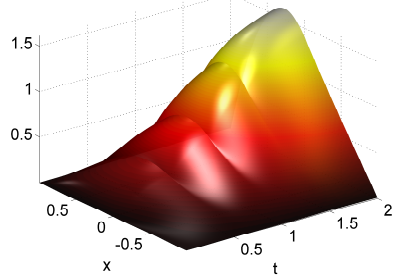

$\theta=5$

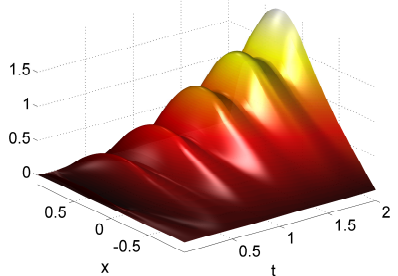

$\theta=0.01$

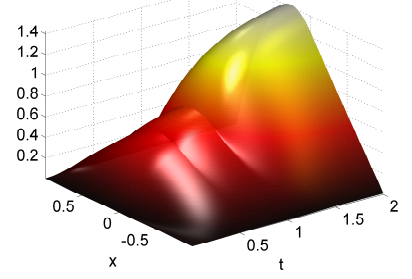

$\theta=20$

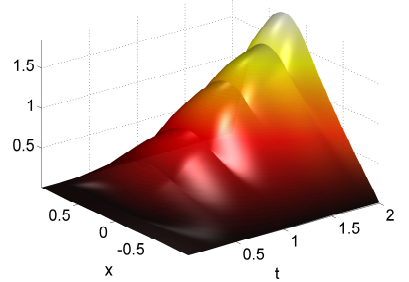

$\theta=1$

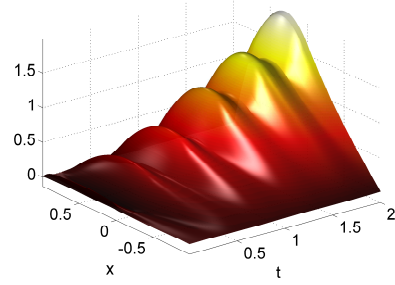

$\theta=0.001$

FIG. 3. Example 1: $\bar{y}_{\sigma}^{\theta}$ for different values of $\theta$. 

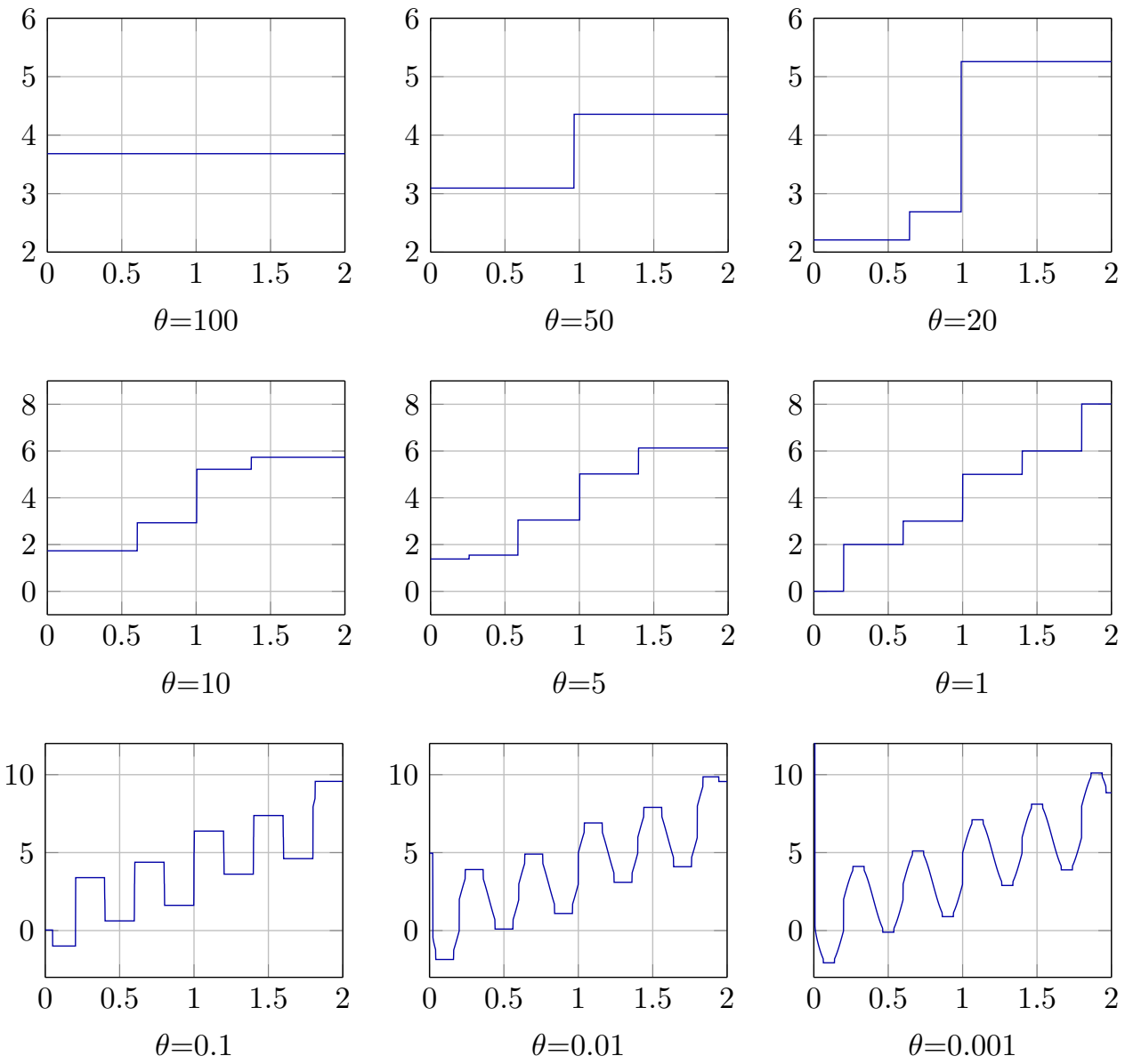

FiG. 4. Example 1: $\bar{u}_{\tau}^{\theta}$ for different values of $\theta$.

7.2. Example 2: Three controls and one spatial dimension. The second example generalizes the first one by allowing for $m \in \mathbb{N}$ controls rather than only one. Moreover, we demonstrate that even in the absence of strict complementarity Algorithm BV yields optimal controls that retain the simple structure of their continuous counterparts. In this example we have $\Omega=(-1,1)$, and $\omega_{j}=\left(a_{j}, b_{j}\right)$ for $1 \leq j \leq m$ with $-1 \leq a_{1}<b_{1} \leq a_{2}<b_{2} \leq \cdots \leq a_{m}<b_{m} \leq 1$. The following construction ensures that for every $j$ the optimal control $\bar{u}_{j}$ has exactly $0 \leq l_{j} \leq m$ jumps and is constant apart from these jumps. We consider

$$
\min _{u \in B V(0, T)^{m}} \frac{1}{2}\left\|y_{u}-y_{d}\right\|_{L^{2}(Q)}^{2}+\sum_{j=1}^{m} \alpha_{j}\left\|u_{j}^{\prime}\right\|_{\mathcal{M}(0, T)},
$$

where $y_{u}$ denotes the solution to (62), but with $u g$ replaced by $\sum_{j=1}^{m} u_{j} g_{j}$. We take $g_{j}=\chi_{\omega_{j}}$ for all $j$. Let $\kappa>0$ and $c_{j k} \geq 0$ for $1 \leq j, k \leq m$. Define

$$
\alpha_{j}=\frac{4 \kappa}{m \pi^{2}}\left(\sin \left(\frac{\pi}{2} b_{j}\right)-\sin \left(\frac{\pi}{2} a_{j}\right)\right) \quad \text { and } \quad \bar{\varphi}(x, t)=\kappa \sin (m \pi t) \cos \left(\frac{\pi}{2} x\right)
$$



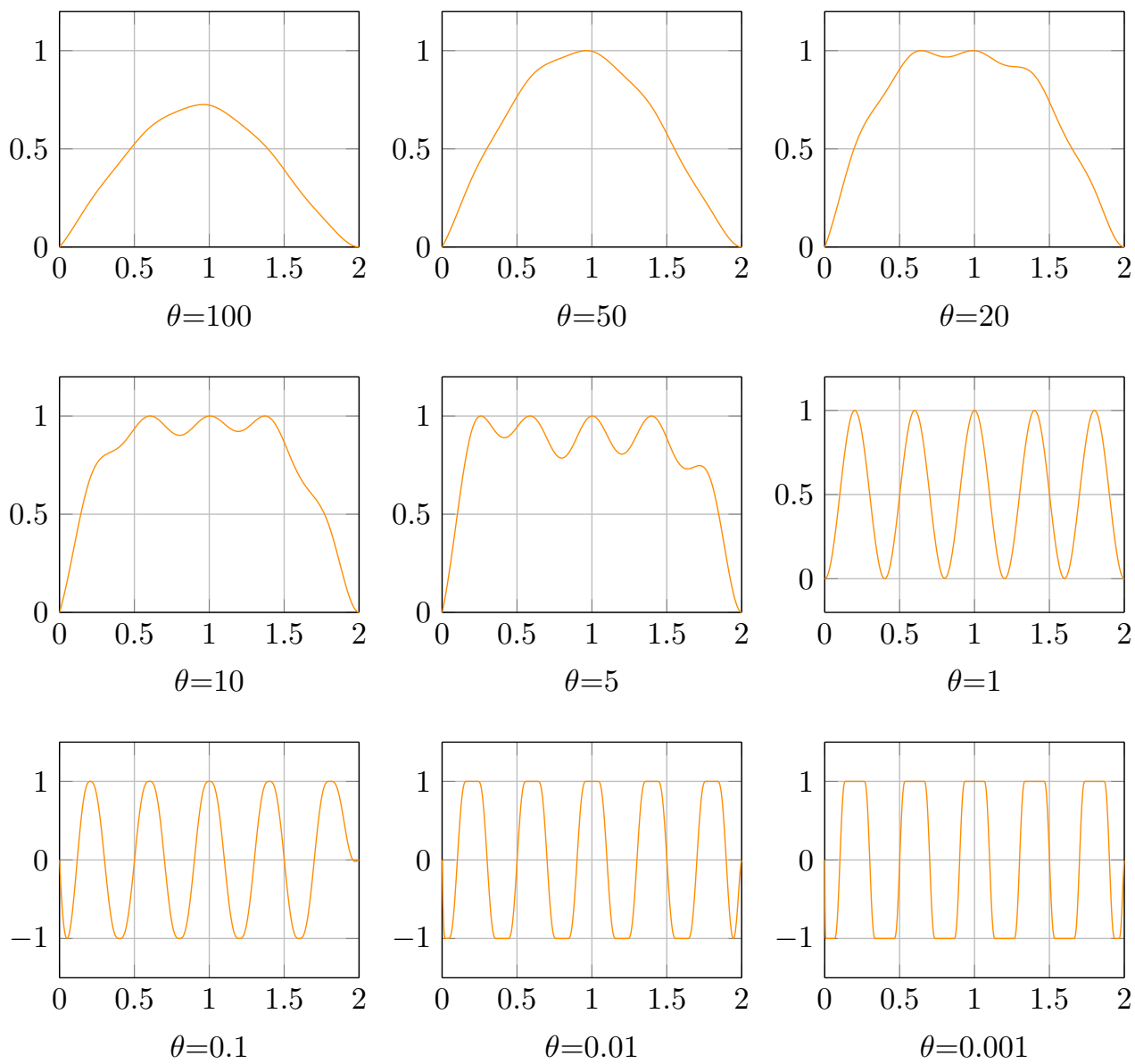

FIG. 5. Example 1: $\bar{\lambda}_{\tau}^{\theta}$ for different values of $\theta$.

as well as for $1 \leq j \leq m$

$$
\bar{u}_{j}=\left\{\begin{array}{ll}
0 & \text { if } t<\frac{1}{m}, \\
c_{j 1} & \text { if } \frac{1}{m}<t<\frac{3}{m}, \\
\vdots & \vdots \\
\sum_{k=1}^{m} c_{j k} & \text { if } \frac{2 m-1}{m}<t<2,
\end{array} \quad \text { and } \quad y_{d}=\bar{y}-L^{*} \bar{\varphi}\right.
$$

where $L=\frac{\partial}{\partial t}-\Delta$ and $\bar{y}=y_{\bar{u}}$. Observing $\bar{\Phi}_{j}(t)=\frac{\alpha_{j}}{2}(1-\cos (m \pi t))$ for all $j$ we readily confirm the optimality of $\bar{u}=\left(\bar{u}_{j}\right)_{j=1}^{m}$ in a similar manner as in the first example.

The numerical results that follow are obtained by choosing $m=3, \omega_{1}=\left(-1,-\frac{1}{2}\right)$, $\omega_{2}=\left(-\frac{1}{4}, \frac{1}{4}\right), \omega_{3}=\left(\frac{1}{2}, 1\right), \kappa=10^{-2}, c_{11}=5, c_{22}=3, c_{33}=1$, and all other $c_{j k}$ equal to zero. This implies that $\bar{u}_{1}, \bar{u}_{2}$, and $\bar{u}_{3}$ each have exactly one jump. These choices are specifically made to study the numerical behavior in situations where the inclusion $\operatorname{supp}\left(\overline{\mathrm{u}}_{\mathrm{j}}^{\prime+}\right) \subset\left\{\mathrm{t} \in[0, \mathrm{~T}]: \bar{\Phi}_{\mathrm{j}}(\mathrm{t})=\alpha_{\mathrm{j}}\right\}$ is strict, which is equivalent to saying that strict complementarity does not hold. Similar to Example 1, we use $y_{\sigma}(\bar{u})$ as a replacement 

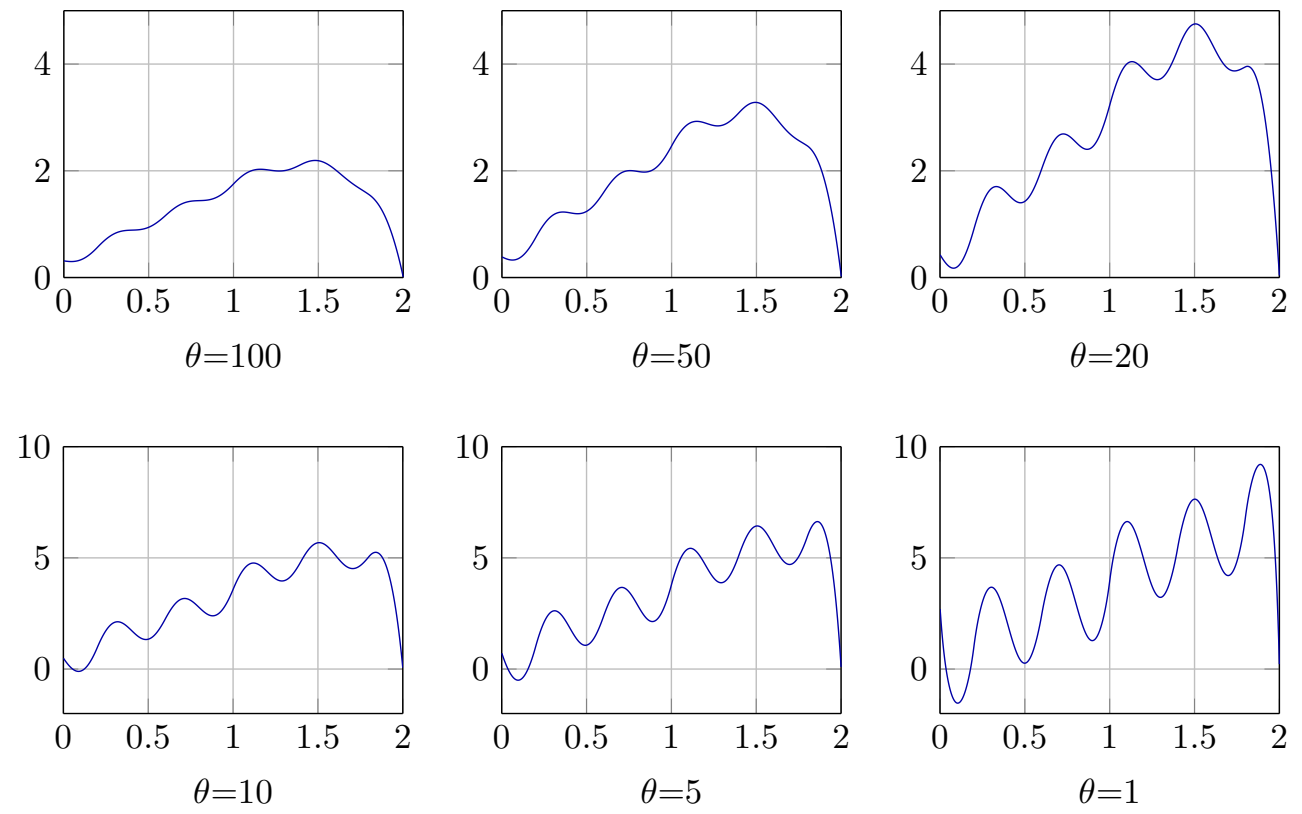

FIG. 6. Example 1: $\bar{u}_{\tau, L^{2}}^{\theta}$ for different values of $\theta$.
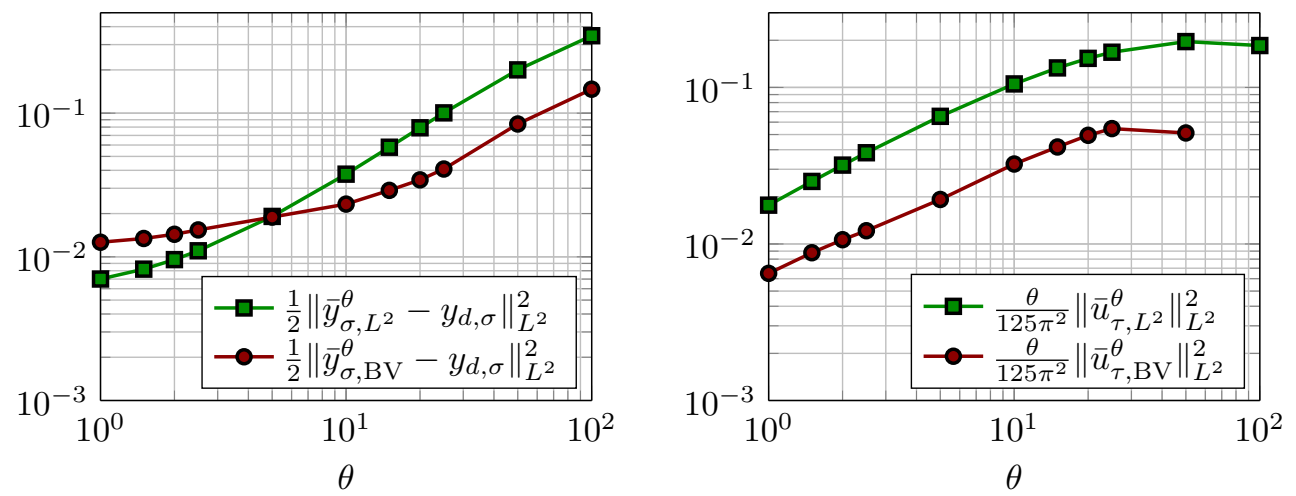

FIG. 7. Example 1: Tracking errors and control norms for $(\mathrm{P})$ and $L^{2}$-regularization.

for $\bar{y}$. We apply Algorithm BV with $N_{t}=6144$ and $N_{h}=255$, which corresponds to $\tau=1 / 3072$ and $h=1 / 128$. Figure 8 displays $y_{d, \sigma}, \bar{y}_{\sigma},\left(\bar{u}_{\tau, j}\right)_{j}$, and $\left(\bar{\lambda}_{\tau, j}\right)_{j}$. The dual variables $\left(\bar{\lambda}_{\tau, j}\right)_{j}$ resemble closely their continuous counterparts $\left(\bar{\lambda}_{j}\right)_{j}=\frac{1}{\alpha_{j}} \bar{\Phi}_{j}=$ $\frac{1}{2}(1-\cos (3 \pi t))$. In particular, each of them has three isolated maximums with value approximately 1 . The approximated optimal controls $\left(\bar{u}_{\tau, j}\right)_{j}$ appear to be very similar to the continuous optimal controls $\left(\bar{u}_{j}\right)_{j}$. In particular, each of these controls exhibits exactly one jump and thus reproduces very well the simple structure of its continuous analogue. Summarizing we conclude from this example and other experiments that the case of strict inclusion $\operatorname{supp}\left(\overline{\mathrm{u}}_{\mathrm{j}}^{\prime}\right) \varsubsetneqq\left\{\mathrm{t} \in[0, \mathrm{~T}]: \bar{\Phi}_{\mathrm{j}}(\mathrm{t})= \pm \alpha_{\mathrm{j}}\right\}$ can be handled very 

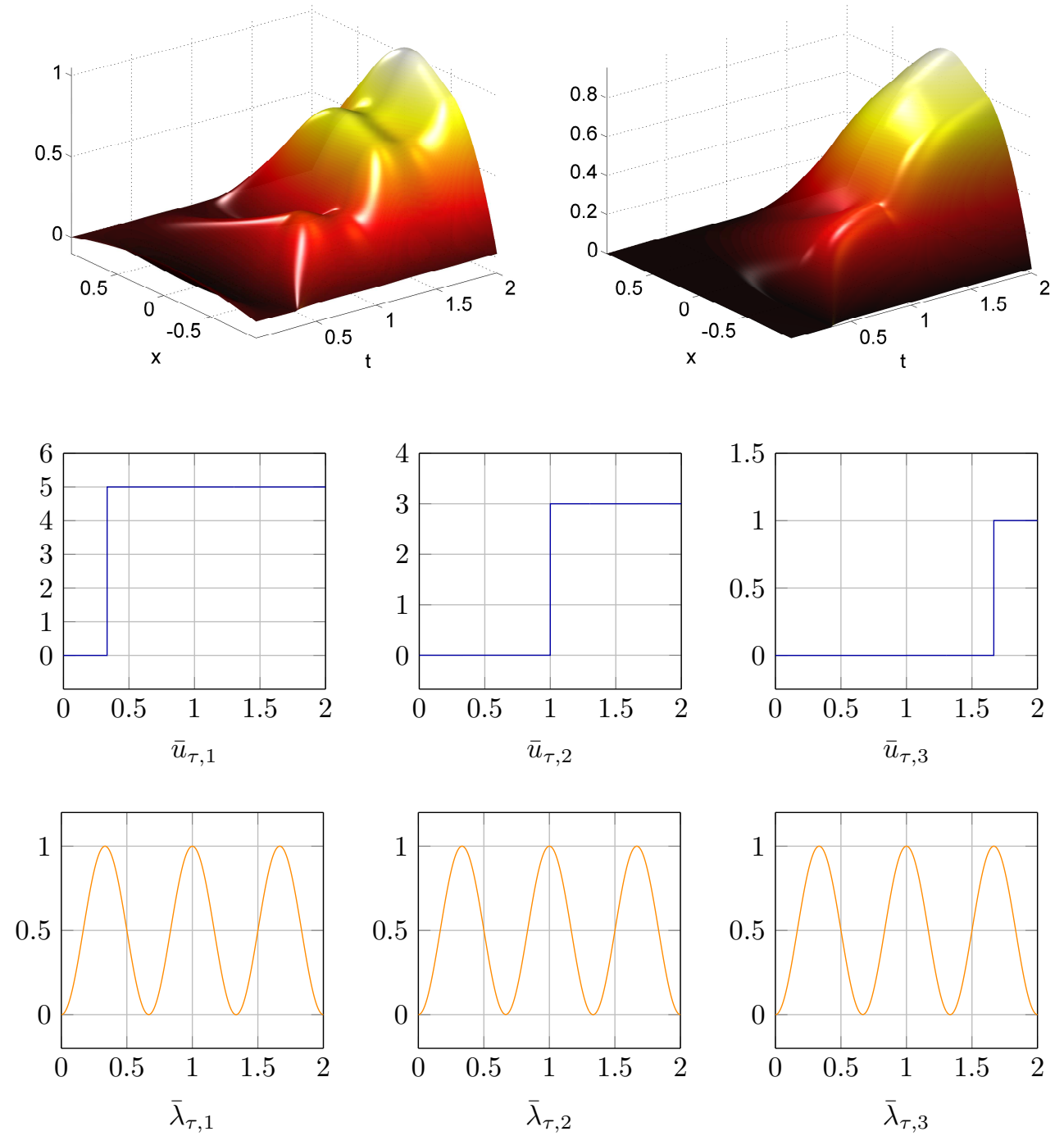

FIG. 8. Example 2: $y_{d, \sigma}$ and $\bar{y}_{\sigma}$ (top left and right), $\left(\bar{u}_{\tau, j}\right)_{j}$, and $\left(\bar{\lambda}_{\tau, j}\right)_{j}$.

well by Algorithm BV.

7.3. Example 3: One control and two spatial dimensions. The first two examples are structurally similar to each other. In particular, in both examples the desired states $y_{d}$ have a rather low temporal regularity. Contrary to this, the third example is constructed in such a way that $y_{d}$ is $C^{\infty}$ with respect to time and space. Moreover, the spatial domain $\Omega$ is two dimensional in this example. In this entirely different setup we will again observe that the optimal control has a very simple structure. We choose $m=1, \Omega=(-1,1)^{2}, \omega=(0,1)^{2}$, and consider the same objective function and state equation as in the first example, except that $\Omega$ and $\omega$ are different. We take $g=\chi_{\omega}, y_{d}\left(x_{1}, x_{2}, t\right)=\left(x_{1}-1.2\right)\left(x_{1}+1\right)\left(x_{2}+1\right)\left(x_{2}-0.9\right) t e^{-t}$, and $\bar{\alpha}=10^{-3}$. The choice of $y_{d}$ yields $\bar{y} \neq y_{d}$ since $y_{d}$ does not satisfy the boundary conditions of 


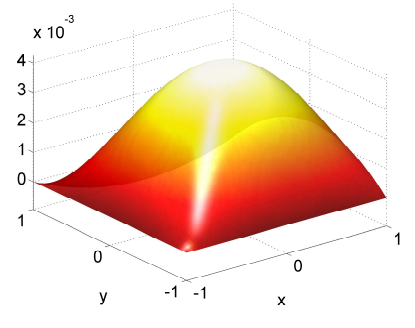

$t=\tau$

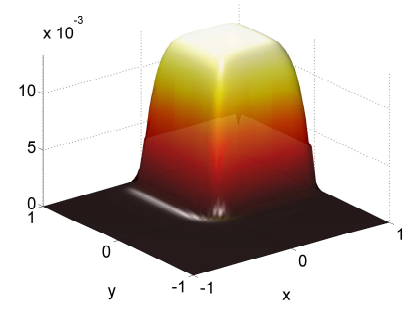

$t=\tau$

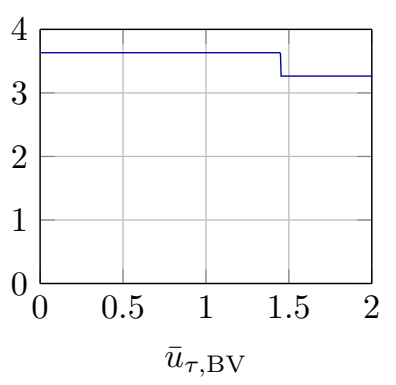

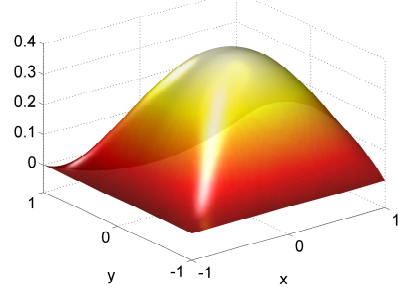

$t=1$

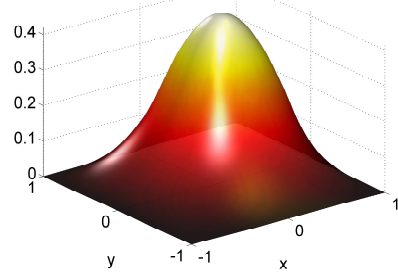

$t=1$

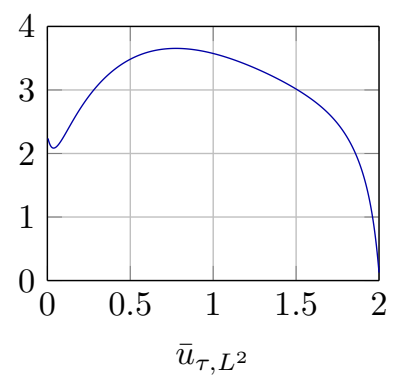

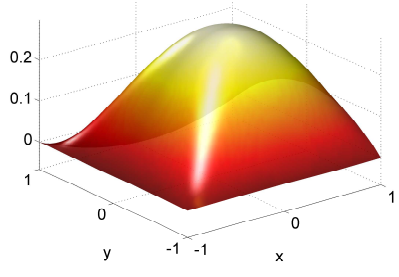

$t=2$

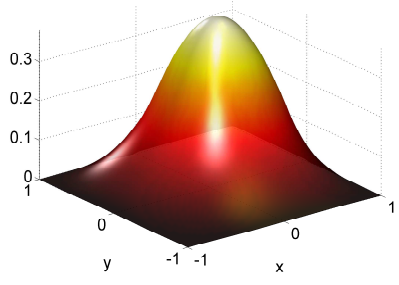

$t=2$

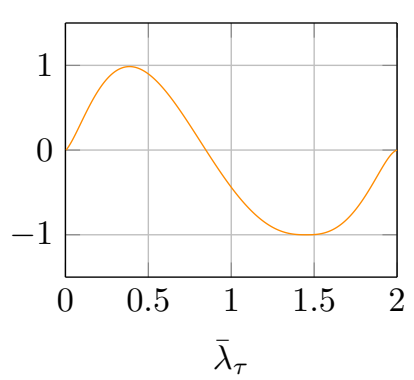

FIG. 9. Example 3: $y_{d, \sigma}$ (top) and $\bar{y}_{\sigma}$ (middle) for different $t, \bar{u}_{\tau, \mathrm{BV}}, \bar{u}_{\tau, L^{2}}$, and $\bar{\lambda}_{\tau}$.

the state equation. We apply Algorithm BV with $N_{t}=512$ and $N_{h}=63^{2}$, which corresponds to $\tau=1 / 256$ and $h=(2-\sqrt{2}) / 64$. Figure 9 shows $y_{d, \sigma}$ and $\bar{y}_{\sigma}$ at different points in time. Moreover, it depicts $\bar{u}_{\tau}=\bar{u}_{\tau, \mathrm{BV}}$ and $\bar{\lambda}_{\tau}$, as well as the optimal control $\bar{u}_{\tau, L^{2}}$ obtained through classical $L^{2}$-regularization (analogously as for Example 1). It seems that in this example $\{t \in[0, T]: \bar{\Phi}(t)= \pm \bar{\alpha}\}$ does not consist of a finite number of points, but has positive measure. However, the structure of $\bar{u}$ is still very simple. In particular, $\bar{u}$ is constant on large parts of its domain.

While the tracking errors associated to the controls in Figure 9 are comparable, $\frac{1}{2}\left\|\bar{y}_{\sigma, \mathrm{BV}}-y_{d, \sigma}\right\|_{L^{2}\left(Q_{h}\right)}^{2} \approx 0.0421$ and $\frac{1}{2}\left\|\bar{y}_{\sigma, L^{2}}-y_{d, \sigma}\right\|_{L^{2}\left(Q_{h}\right)}^{2} \approx 0.0422$, the structure of the $\mathrm{BV}$-control is simpler than that of the $L^{2}$-control. For the control terms in the objectives we have $\bar{\alpha}\left\|\left(\bar{u}_{\tau, \mathrm{BV}}\right)^{\prime}\right\|_{\mathcal{M}(0, T)} \approx 4 \cdot 10^{-4}$ and $\frac{\bar{\alpha}}{2}\left\|\bar{u}_{\tau, L^{2}}\right\|_{L^{2}(0, T)}^{2} \approx 1 \cdot 10^{-2}$.

8. Conclusions. In this paper we gave a rather complete analysis for optimal control problems governed by semilinear parabolic equations for the case where the temporal control cost is realized in the BV-seminorm. This leads to optimal controls 
that are piecewise constant in time. This simple structure of the optimal controls, which is confirmed analytically and numerically, is desirable from a practical point of view. It is distinctly different from optimal controls that arise from quadratic controlcost functionals. The obtained results can be expanded in several directions. For instance, it would be interesting to consider controls that are BV functions in space and time, or to use BV functionals in the context of switching controls.

\section{REFERENCES}

[1] L. Ambrosio, N. Fusco, and D. Pallara, Functions of Bounded Variation and Free Discontinuity Problems, Oxford University Press, New York, 2000.

[2] E. CASAs, Pontryagin's principle for state-constrained boundary control problems of semilinear parabolic equations, SIAM J. Control Optim., 35 (1997), pp. 1297-1327, https://doi.org/ $10.1137 /$ S0363012995283637.

[3] E. Casas, C. Clason, and K. Kunisch, Approximation of elliptic control problems in measure spaces with sparse solutions, SIAM J. Control Optim., 50 (2012), pp. 1735-1752, https: //doi.org/10.1137/110843216.

[4] E. Casas, C. Clason, And K. Kunisch, Parabolic control problems in measure spaces with sparse solutions, SIAM J. Control Optim., 51 (2013), pp. 28-63, https://doi.org/10.1137/ 120872395.

[5] E. Casas, P. Kogut, and G. Leugering, Approximation of optimal control problems in the coefficient for the p-Laplace equation. I. Convergence result, SIAM J. Control Optim., 54 (2016), pp. 1406-1422, https://doi.org/10.1137/15M1028108.

[6] E. CASAS AND K. Kunisch, Optimal control of semilinear elliptic equations in measure spaces, SIAM J. Control Optim., 52 (2014), pp. 339-364, https://doi.org/10.1137/13092188X.

[7] E. CASAs And F. TröLtzsch, Second order analysis for optimal control problems: Improving results expected from abstract theory, SIAM J. Optim., 22 (2012), pp. 261-279, https: //doi.org/10.1137/110840406.

[8] E. Casas And F. Tröltzsch, Second-order optimality conditions for weak and strong local solutions of parabolic optimal control problems, Vietnam J. Math., 44 (2016), pp. 181-202, https://doi.org/10.1007/s10013-015-0175-6.

[9] P. Ciarlet, The Finite Element Method for Elliptic Problems, North-Holland, Amsterdam, 1978, https://doi.org/10.1002/nme.1620140514.

[10] C. Clason and K. Kunisch, A duality-based approach to elliptic control problems in nonreflexive Banach spaces, ESAIM Control Optim. Calc. Var., 17 (2011), pp. 243-266, https: //doi.org/10.1051/cocv/2010003.

[11] I. Ekeland and R. TÉmam, Convex Analysis and Variational Problems, Unabridged, corrected republication of the 1976 English original, Classics Appl. Math. 28, SIAM, Philadelphia, 1999, https://doi.org/10.1137/1.9781611971088.

[12] E. Giusti, Minimal Surfaces and Functions of Bounded Variation, Monogr. Math. 80, Birkhäuser Verlag, Basel, 1984, https://doi.org/10.1007/978-1-4684-9486-0.

[13] D. Hafemayr and B. Vexler, Optimale Steuerung von Differentialgleichungen mit BVFunktionen, TU Munich, Munich, Germany, 2016.

[14] R. Herzog, G. Stadler, and G. Wachsmuth, Directional sparsity in optimal control of partial differential equations, SIAM J. Control Optim., 50 (2012), pp. 943-963, https: //doi.org/10.1137/100815037.

[15] K. Kunisch, K. Pieper, And B. Vexler, Measure valued directional sparsity for parabolic optimal control problems, SIAM J. Control Optim., 52 (2014), pp. 3078-3108, https://doi. org/10.1137/140959055.

[16] D. Meidner and B. Vexler, A priori error estimates for the space-time finite element discretization of parabolic optimal control problems Part I: Problems without control constraints, SIAM J. Control Optim., 47 (2008), pp. 1150-1177, https://doi.org/10.1137/ 070694016 .

[17] I. Neitzel And B. Vexler, A priori error estimates for space-time finite element discretization of semilinear parabolic optimal control problems, Numer. Math., 120 (2012), pp. 345-386, https://doi.org/10.1007/s00211-011-0409-9.

[18] K. PiePer ANd B. VeXler, A priori error analysis for discretization of sparse elliptic optimal control problems in measure space, SIAM J. Control Optim., 51 (2013), pp. 2788-2808, https://doi.org/10.1137/120889137.

[19] P. RAVIART AND J. Thomas, Introduction à l'analyse numérique des equations aux dérivées partielles, Collect. Math. Appl. Matrise, Masson, Paris, 1983. 
[20] W. Rudin, Real and Complex Analysis, McGraw-Hill, London, 1970.

[21] R. Showalter, Monotone Operators in Banach Space and Nonlinear Partial Differential Equations, Math. Surveys Monogr. 49, American Mathematical Society, Providence, RI, 1997, https://doi.org/10.1090/surv/049.

[22] V. Thомée, Galerkin Finite Element Methods for Parabolic Problems, 2nd ed., Springer Ser. Comput. Math. 25, Springer-Verlag, Berlin, 2006, https://doi.org/10.1007/3-540-33122-0.

[23] F. Tröltzsch, Optimal Control of Partial Differential Equations: Theory, Methods and Applications, Grad. Stud. Math. 112, American Mathematical Society, Providence, RI, 2010, https://doi.org/10.1090/gsm/112.

[24] M. Ulbrich, Semismooth Newton Methods for Variational Inequalities and Constrained Optimization Problems in Function Spaces, MOS-SIAM Ser. Optim. 11, SIAM, Philadelphia, 2011, https://doi.org/10.1137/1.9781611970692. 